\title{
Prandtl's Secondary Flows of the Second Kind. Problems of Description, Prediction, and Simulation
}

\author{
N. V. Nikitin ${ }^{a, *}$, N. V. Popelenskayaa ${ }^{a, * *}$, and A. Stroh ${ }^{b, * * *}$ \\ ${ }^{a}$ Institute of Mechanics, Moscow State University, Michurinskii pr. 1, Moscow, 119192 Russia \\ ${ }^{b}$ Institute of Fluid Mechanics, Karlsruhe Institute of Technology, Karlsruhe, Germany \\ *e-mail:nvnikitin@mail.ru \\ **e-mail: aero.natap@mail.ru \\ ***e-mail: alexander.stroh@kit.edu
}

Received March 23, 2021; revised March 25, 2021; accepted March 25, 2021

\begin{abstract}
The occurrence of turbulent pulsations in straight pipes of noncircular cross-section leads to the situation, when the average velocity field includes not only the longitudinal component but also transverse components that form a secondary flow. This hydrodynamic phenomenon discovered at the twenties of the last century (J. Nikuradse, L. Prandtl) has been the object of active research to the present day. The intensity of the turbulent secondary flows is not high; usually, it is not greater than $2-3 \%$ of the characteristic flow velocity. Nevertheless, their contribution to the processes of transverse transfer of momentum and heat is comparable to that of turbulent pulsations. In this paper, a review of experimental, theoretical, and numerical studies of secondary flows in straight pipes and channels is given. Emphasis is placed on the issues of revealing the physical mechanisms of secondary flow formation and developing the models of the apriori assessment of their forms. The specific features of the secondary flow development in open channels and channels with inhomogeneously rough walls are touched upon. The approaches of semiempirical simulation of turbulent flows in the presence of secondary flows are discussed.
\end{abstract}

Keywords: turbulent flows in straight pipes, secondary flows, Navier-Stokes equations, direct numerical simulation, rough walls, RANS modeling

DOI: $10.1134 / \mathrm{S} 0015462821040091$

One of the interesting and practically important manifestations of turbulence is the capability to induce the so-named secondary flows, that is, organized fluid motions in the plane perpendicular to the main stream direction. The most known in this respect are flows in straight pipes of noncircular cross-section. Fairly far from the entry laminar flows in these pipes are one-dimensional, that is, at any point the fluid velocity is directed along the pipe. Contrariwise, in turbulent regimes the average velocity field contains nonzero transverse components. The first experimental evidence of the generation of turbulent secondary flows was obtained by Nikuradse in pipes with rectangular and triangular cross-sections [1]. He found that the isotachs (contours of the mean longitudinal velocity in the cross-sectional plane) have unusual convexities directed toward the corners (Fig. 1). Prandtl supposed that the characteristic distortions of the isotachs are caused by secondary flows induced by turbulent pulsations. The fluid flows along the bisector toward the corner and spreads on both sides along the walls. Thus, the fluid particles with a greater momentum are transferred from the flow core to the walls, into the corner region, which gives rise to an anomalous velocity increase in this region of the pipe [2-4] (Fig. 2). Prandtl's supposition on the occurrence of secondary flows in straight noncircular pipes was confirmed in [5] by mean velocity measurements and turbulent flow visualization in pipes with different cross-sections. In those times the secondary flow intensity could not be reliably measured. The first direct measurements of the secondary flows in rectangular pipes were carried out only 30 years later [6]. Secondary flows in straight pipes occur only in turbulent flows. Prandtl [7, 8] proposed to call these flows the secondary flows of the second kind, as distinct from the secondary flows of the first kind occurring within curved flows under the action of centrifugal effects in both turbulent and laminar flows. The intensity of the secondary flows of the first kind can be as high as tens of percents of the main stream velocity. The characteristic velocities of the secondary flows of the second kind are considerably lower. In pipes they are usually not greater than $2-3 \%$. None- 


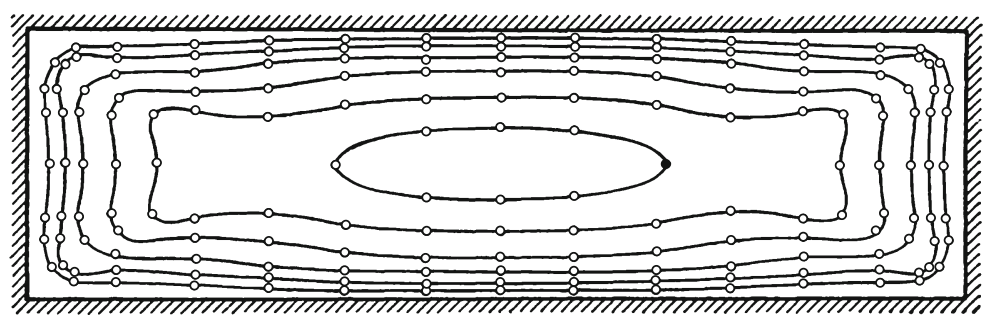

Fig. 1. Mean velocity contours in the cross-section of a rectangular pipe [1, 4].
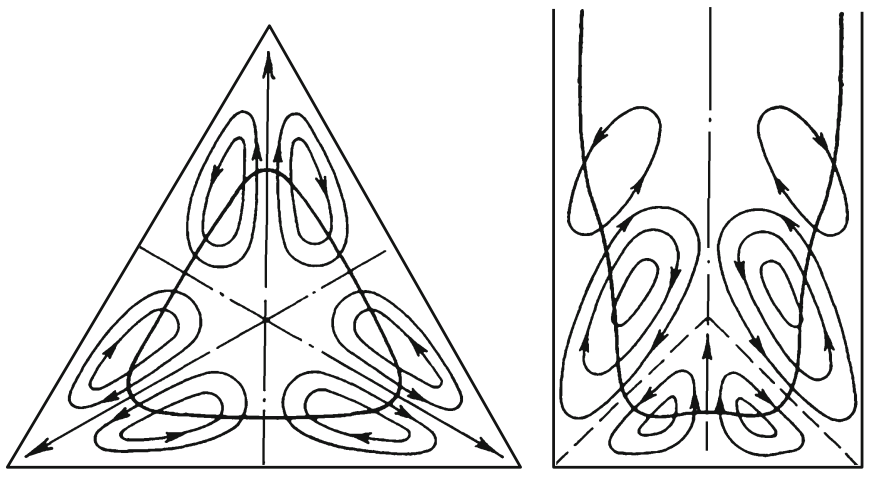

Fig. 2. Schematic of secondary flows proposed by Prandtl [2, 4].

theless, their contribution to the processes of the transverse transport is comparable to that of turbulent pulsations [9].

In [10] a somewhat different principle of secondary flow classification was proposed. The flows of the first type include those, where the longitudinal component of the mean vorticity is formed as a result of a variation in the mean vorticity vector direction. In particular, this can occur in pipe bends, independent of the flow nature, be it laminar or turbulent. In turbulent flows the Reynolds stresses make the contribution to the formation of the mean vorticity but in these cases their effect reduces to the secondary flow attenuation at the expense of turbulent diffusion. In the flows of the second type precisely the Reynolds stresses are the cause of the mean longitudinal vorticity generation. In [10] a short review of the secondary flows of the first and second types was given. In [11, 12] the special features of secondary flows in threedimensional turbulent flows in corner regions are described, including the cases of developing and transitional flows. This study presents the review and analysis of the results of investigations of secondary flows occurring in long straight pipes of noncircular cross-section. Apart from the original results of different authors, certain results obtained by the authors of this study are also presented.

\section{SECONDARY FLOWS IN PIPES OF DIFFERENT CROSS-SECTIONS}

There has been much research devoted to the elicitation of the physical mechanisms and the explanation of the causes of the generation of the secondary flows of the second kind. Prandtl supposed that turbulent pulsations in the corner region of the pipe cross-section take place not only in the direction of the mean velocity gradient but also, and at a greater intensity, in the transverse direction, that is, along isotachs. When fluid particles move along curved isotachs, there arises a force directed toward their convexity, which ultimately leads to the secondary flow generation. Later, in book [13] he formulated the principle, in accordance with which the secondary flows in noncircular pipes are directed from the walls into the flow in the locations of increased friction stress on the wall and, contrariwise, outward from the main stream to the walls in the locations of lowered friction, in particular, in the corner regions of the crosssection. Thus, the friction is equalized along the pipe cross-section perimeter. In the experiment [6] the secondary flows in the pipes of rectangular cross-section were studied for several rectangle aspect ratios. It was shown that the friction variation along the boundary is consistent with the secondary flow direction, which confirms the conclusions made in [13]. Another principle proposed in [14, 15] is based on an anal- 
ysis of the terms of the balance equation for the kinetic energy of turbulence. If at certain location of the flow the kinetic energy production is considerably greater than viscous dissipation, then there arises a secondary flow transferring the fluid particles with a greater kinetic energy from this region toward the regions, where the energy production is inferior than dissipation.

The secondary flow in a cross-sectional plane is uniquely determined from the distribution of the longitudinal vorticity component of the mean flow $\Omega_{x}=\partial W / \partial y-\partial V / \partial z$. Here and in what follows, $(x, y, z)$ are the Cartesian coordinates with the $x$ axis aligned with the pipe axis and $(u, v, w)$ and $\left(\omega_{x}, \omega_{y}, \omega_{z}\right)$ are the corresponding components of the velocity $\mathbf{u}$ and vorticity $\nabla \times \mathbf{u}$ vectors. The capital letters denote the mean values, while the primes relate to pulsations: $q(t, x, y, z)=Q(y, z)+q^{\prime}(t, x, y, z)$ for an arbitrary variable $q$; the statistic averaging is denoted by the angular brackets: $Q=\langle q\rangle$. The equation for $\Omega_{x}$ in a turbulent flow was derived in [16]

$$
V \frac{\partial \Omega_{x}}{\partial y}+W \frac{\partial \Omega_{x}}{\partial z}-v\left(\frac{\partial^{2} \Omega_{x}}{\partial y^{2}}+\frac{\partial^{2} \Omega_{x}}{\partial z^{2}}\right)=S
$$

The fluid is assumed to be incompressible and the flow is time-independent and uniform in the longitudinal direction. Thus, the mean values do not depend on time and the longitudinal coordinate. The first two terms on the left side of Eq. (1.1) govern the longitudinal vorticity convection by the secondary flow. Their effect consists in the equalization of vorticity values along the trajectory. The third term corresponds to viscous effects smoothing the gradients $\Omega_{x}$. The source term on the right side of Eq. (1.1) is responsible for the production of the longitudinal vorticity; it is expressed in terms of the gradients of the Reynolds stresses, as follows:

$$
S=\frac{\partial^{2}}{\partial y \partial z}\left(\left\langle v^{\prime 2}\right\rangle-\left\langle w^{\prime 2}\right\rangle\right)+\left(\frac{\partial^{2}}{\partial z^{2}}-\frac{\partial^{2}}{\partial y^{2}}\right)\left\langle v^{\prime} w^{\prime}\right\rangle \equiv S_{1}+S_{2} .
$$

As shown in [17, 18], Eq. (1.1) with zero right side and the no-slip conditions imposed on fixed walls does not have nontrivial solutions. Therefore, in steady laminar flows in straight pipes secondary flows cannot occur. On the other hand, it is obvious that at $S \neq 0$ the absence of a secondary flow is impossible. As shown in [16], the source term in Eq. (1.1) is nonzero in the corner regions of pipes of rectangular crosssection, which explains the generation of secondary flows. From the considerations of symmetry it follows that $S$ inverses sign on any line of symmetry. Thus, the lines of symmetry in the pipe cross-section separate the regions of opposite circulation [19].

The two terms in Eq. (1.2) are torques acting on the fluid. To make an estimate of these terms, in experiment [19] all the three velocity components and six components of the Reynolds stress tensor were measured in a pipe of square cross-section. It was concluded that, when the coordinate axes $(y, z)$ are parallel to the walls, the first term $S_{1}$ containing the difference of the normal stresses $\left\langle v^{2}\right\rangle-\left\langle w^{2}\right\rangle$ predominates over the second term $S_{2}$ containing the derivatives of the shear stress $\left\langle v^{\prime} w^{\prime}\right\rangle$. Thus, the longitudinal vorticity is produced by the term $S_{1}$ and transferred into locations, where it is dissipated under the action of viscosity. The remark concerning the orientation of the coordinate axes is essential, since $S_{1}$ and $S_{2}$ are not invariant with respect to variations of the coordinate system. In particular, upon rotation of the $(y, z)$ axes by an angle of $45^{\circ}$ the values of $S_{1}$ and $S_{2}$ change places. Another interpretation of the fact that the secondary flows in a square pipe are induced by the normal stress gradients was proposed in [20]. It was shown that the Reynolds equations in the cross-sectional plane of the pipe with discarded shear stress gradients have a solution only in the presence of a secondary flow directed along the walls, outward from the corner. The fact that neglecting the Reynolds shear stress $\left\langle v^{\prime} w^{\prime}\right\rangle$ leads to the conclusion about the necessary presence of a secondary flow in the pipe of square cross-section can also be seen from Eq. (1.2). It can easily be shown that $S_{1}$ is not identically zero and, therefore, in the absence of the compensating second term $S$ also is not identically zero and Eq. (1.1) has a nonzero solution. In [21] it was asserted that the inequality $\left\langle v^{\prime 2}\right\rangle-\left\langle w^{\prime 2}\right\rangle \neq 0$ is the sufficient condition for the generation of a secondary flow in pipes of noncircular cross-section. On the one hand, both this inequality and secondary flows take place in most of noncircular pipes. On the other hand, in circular pipes or, for example, in pipes of annular cross-section secondary flows do not exist despite the inequality of the normal stresses, while the reasoning presented in [21] does not use the condition of the pipe cross-section non-circularity.

The accuracy of the results and the conclusions concerning the $S_{1}$ predominance in Eq. (1.2) made in [19] were brought into question in [22]. It was shown that errors on the $1 \%$ level in the hot-wire ane- 
mometer signal in the measurement system used can lead to $100 \%$ errors in evaluating the shear stresses $\left\langle v^{\prime} w^{\prime}\right\rangle$. More accurate measurements [23] showed that the Reynolds stress gradients generating the terms $S_{1}$ and $S_{2}$ in Eq. (1.2) are similar in value, whereas the convective and viscous terms are two orders less. It was concluded that the secondary flow arises under the action of a small difference between the gradients of the pressure and the Reynolds stresses. On the basis of an analysis of experimental studies in rectangular pipes made in the years 1960-1970 in [24] the following conclusions were made. The terms in Eq. (1.2) are similar in value but their signs are opposite. Their amplitudes are considerably greater than the values of the convective terms (1.1). The viscous terms are negligibly small everywhere, except for the immediate vicinity of a corner. Thus, the production of the longitudinal vorticity is described by a small difference of relatively large quantities $S_{1}$ and $S_{2}$.

From the end of the eighties of the last century numerical simulation has become an equal instrument in investigating turbulence. In calculating the secondary flows the eddy-resolving methods of solving the Navier-Stokes equations are used, namely, Large Eddy Simulation (LES) and Direct Numerical Simulation (DNS). In the former approach the calculations are performed on a relatively coarse grid, while the small-scale components are taken into account using certain models for describing small (subgrid) scales. In the latter approach all important scales of the turbulent motion are resolved on the computational grid. The first detailed calculations of turbulent flows in a pipe of square cross-section were carried out in [25] (LES) and [26, 27] (DNS). Although in calculations the Reynolds numbers are usually considerably lower than in most of experiments, the results of the calculations made it possible to considerably broaden the general knowledge of the properties of flows in noncircular pipes and, in particular, to deepen the understanding of the mechanisms of secondary flow generation. In [26] the calculations were performed at the Reynolds number $\mathrm{Re}=4410$ based on the mean velocity $U_{b}$ and the pipe width $2 h$. For the first time, the distributions of the mean and fluctuation parameters in the pipe cross-section were determined with a high accuracy. The mean velocity distribution and the secondary flow streamlines obtained for this Reynolds number in [28] are presented in Fig. 3. In this figure and everywhere in what follows the blue and red colors correspond to small and large values of the parameters presented, respectively. The isolines with negative values are presented by broken curves. The solid streamlines of the secondary flow correspond to counterclockwise fluid motion, while the dashed ones relate to the clockwise motion. In the vicinity of each of the four corners of the pipe cross-section the fluid particles are transferred by the secondary flow from the pipe center toward the corners along the bisectors and spread on both sides along the walls. A maximum velocity in the secondary flow (on the corner bisectors and in the wall region) is $0.02 U_{b}$. The secondary flows exhibit themselves as characteristic convexities of the mean velocity contours directed toward the corner points. In Fig. 4 we have plotted the distributions of the mean longitudinal vorticity $\Omega_{x}(y, z)$ and the source term $S(y, z)$ on the right side of Eq. (1.1) determining the $\Omega_{x}$ production. A quarter of the pipe cross-section is presented. Here, all the parameters are normalized by the viscous scales of the velocity $U_{\tau}=\sqrt{\tau_{w} / \rho}$ and the length $l_{\tau}=v / U_{\tau}$, where $\tau_{w}$ is the mean friction on the pipe wall and $\rho$ and $v$ are the fluid density and viscosity. The normalization by the viscous (near-wall) scales is denoted by the superscript ' + '. The Reynolds number $\operatorname{Re}_{\tau}=U_{\tau} h / v \equiv h^{+}$. The vorticity production is concentrated in the wall region of the corner domain. A maximum of $|S|$ is reached at points $\left(y^{+}, z^{+}\right) \approx(35,10)$ and $(10,35)$, symmetric relative to the corner bisector. The results of calculations [26] put an end of the discussion concerning the relative importance of the terms $S_{1}$ and $S_{2}$ in expression (1.2) for the source term $S$ of Eq. (1.1). The distributions of $S_{1}$ and $S_{2}$ are plotted in Fig. 5. At the points of $|S|$ maximum the source term is by $90 \%$ determined by the term $S_{1}$ but nearer to the wall the absolute values of each of these terms are twice as large as the values of the $|S|$ maximum and by $90 \%$ balance against one another. Thus, the essential importance of both terms entering into expression (1.2) is confirmed. Contrary to the conclusions made in [24], in [26] it was shown that the convective terms in Eq. (1.1) are negligibly small compared with the viscous terms which balance the terms responsible for $\Omega_{x}$ production.

Analogous conclusions were made in [27] for $\mathrm{Re} \approx 10^{4}$. In that study, the quadrantal analysis of the shear stresses $\left\langle v^{\prime} w^{\prime}\right\rangle$ was also carried out. It was attempted to relate the appearance of secondary flows with the interaction of intense turbulent ejections in-between neighboring walls in the corner region. In [29] certain details of the statistical characteristics with variation in the Reynolds number are refined. It was shown that at different distances from the corner the mean velocity profiles satisfy the wall law, when normalized by the local wall friction. In [30] it was found that the coordinates of local extrema of $\Omega_{x}$ and the streamlines of the secondary flow $\Psi$ in the cross-sectional plane, which approximately coincide at the 


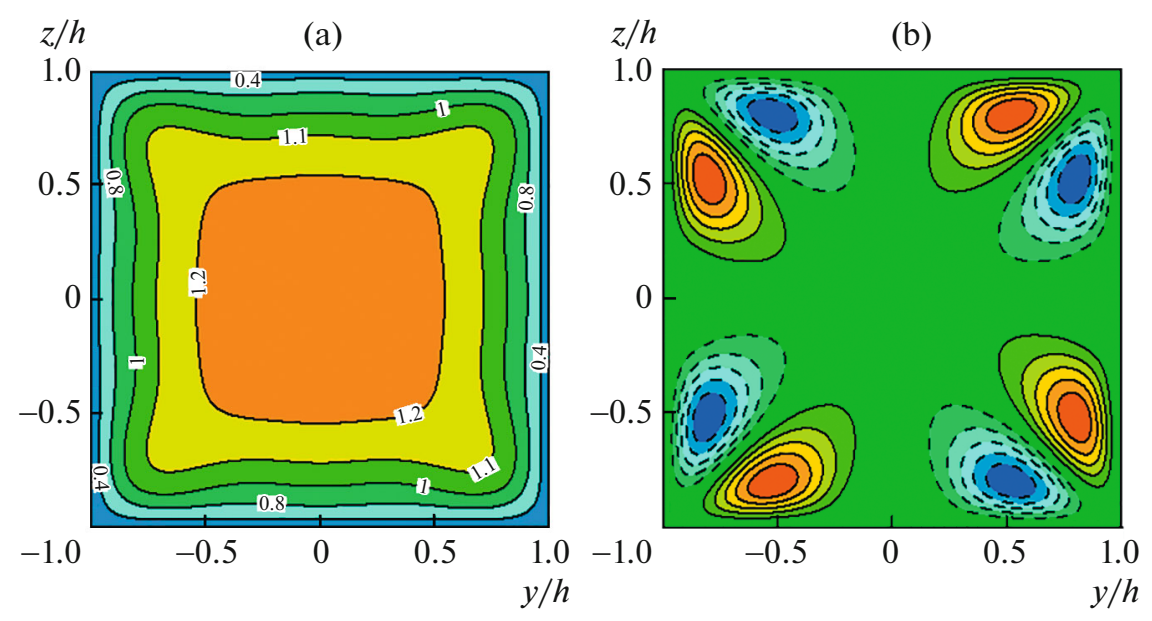

Fig. 3. Mean velocity distribution $U / U_{b}$ (a) and secondary flow streamlines (stream function contours $\Psi h / U_{b}= \pm 0.0004 n, n=1-6$ ) (b) in the cross-section of a square pipe at $\operatorname{Re}=4410$ [26, 28].

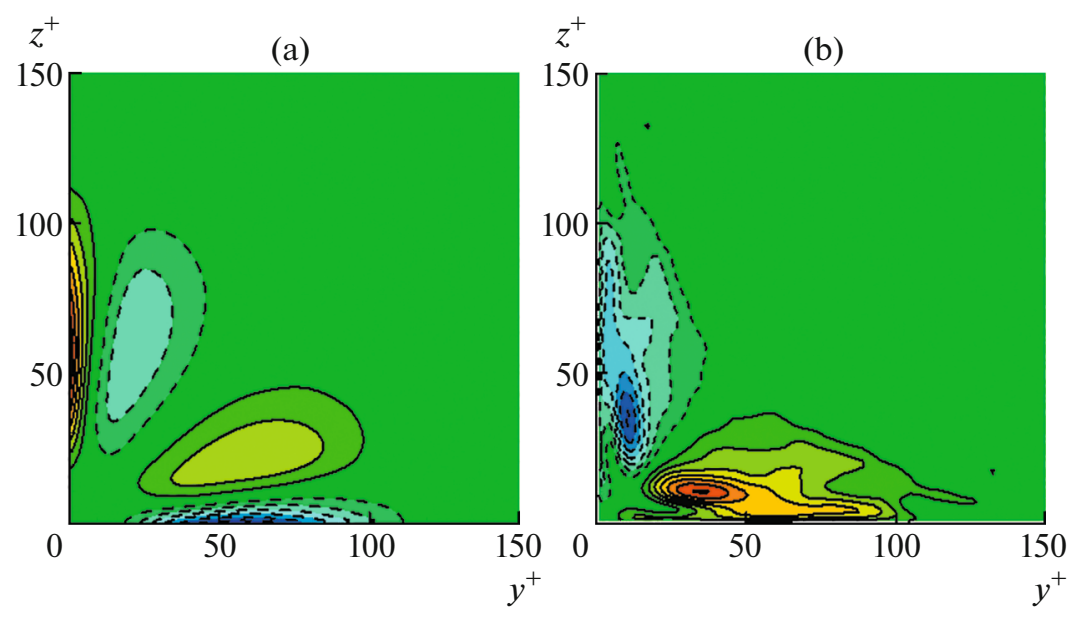

Fig. 4. Distributions of the mean longitudinal vorticity $\Omega_{x}^{+}= \pm 0.01 n, n=1-6$ (a) and the source term in Eq. (1.1) $S^{+}= \pm 0.0001 n, n=1-7(b)$ in the cross-section of a square pipe at $\operatorname{Re}=4410\left(\operatorname{Re}_{\tau}=150\right)[26,28]$.

lowest Reynolds numbers $\mathrm{Re} \sim 2000$, diverge at greater Re values. In this case, the coordinates of local extrema of $\Omega_{x}$ no longer vary with increase in Re, when expressed in viscous units. On the contrary, the coordinates of extrema of $\Psi$ remain constant on the global scale. A certain statistical relationship between the secondary flow and turbulent near-wall structures, such as streaky structures occurring in the wall region of a turbulent boundary layer, can be observable. The streaky structures that are closest to the corner have predominantly an elevated velocity and are formed at a distance of 50 viscous lengths. In this case, the sign of the quasilongitudinal vortices in the wall region predominantly coincides with the sign of $\Omega_{x}$. In [31], where LES calculations were performed for three Reynolds numbers, $\operatorname{Re}_{\tau}=190,300,550$ $(\mathrm{Re} \approx 6000-20000)$, it was concluded that, when normalized by the viscous scales, the values and distributions of the terms corresponding to viscous diffusion and the longitudinal vorticity production in Eq. (1.1) do not change with variation in Re. DNS calculations were carried out in [32] up to $\operatorname{Re}_{\tau}=600$ $(\mathrm{Re} \approx 21400)$. Emphasis was placed on the refinement of the distributions of the mean velocity components and the pulsation intensities. The calculations up to even greater values $\operatorname{Re} \approx 40000$ were performed in [33]. It was shown that outside the corner region the secondary flows can be at a good accuracy approximated by the eigenfunctions of the Laplace operator. It was concluded that at high Reynolds numbers the effect of the secondary flows on the integral properties of the flow is only slight. 


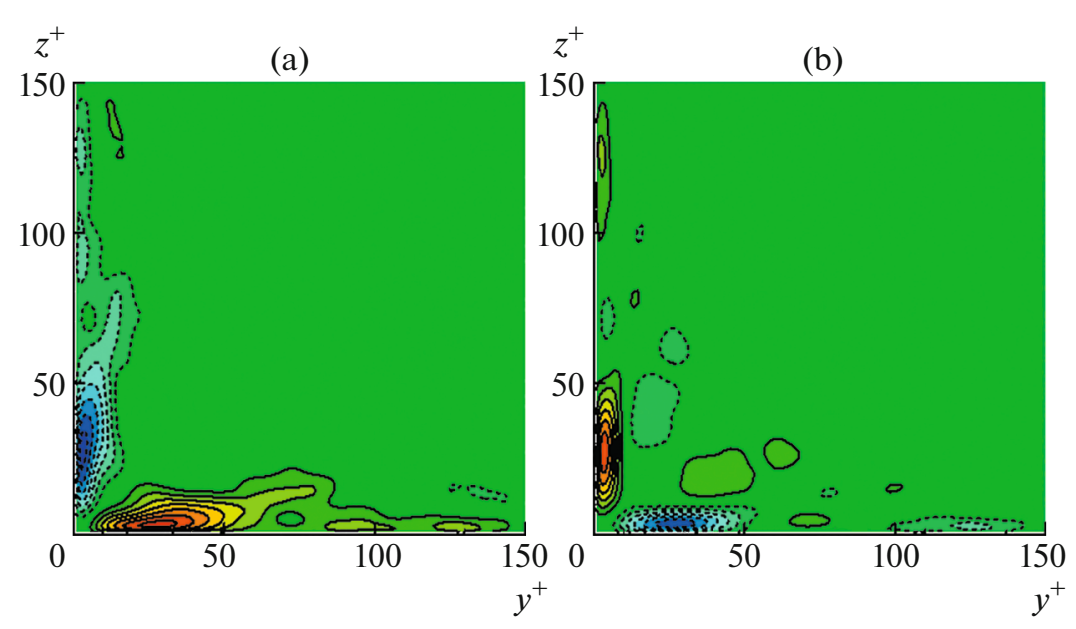

Fig. 5. Distributions of the quantities $S_{1}$ and $S_{2}$ (1.2) in the cross-section of a square pipe at $\operatorname{Re}=4410\left(\operatorname{Re}_{\tau}=150\right)$ [26, 28]; $S_{1}^{+}= \pm 0.0002 n, n=1-7$ (a) and $S_{2}^{+}= \pm 0.0002 n, n=1-6$ (b).
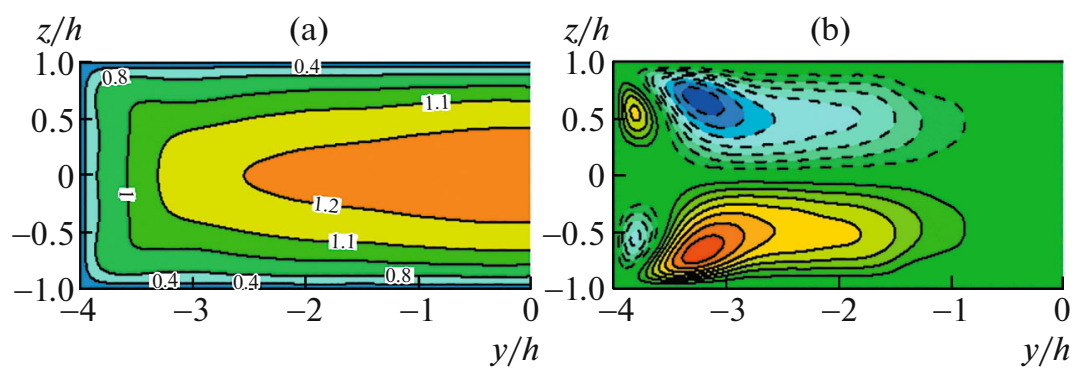

Fig. 6. Distribution of the mean velocity $U / U_{b}$ (a) and secondary flow streamlines (stream function contours $\Psi h / U_{b}= \pm 0.0005 n, n=1-8$ ) (b) in the cross-section of a rectangular pipe with the aspect ratio 4:1 at $\operatorname{Re}=4410$ [34].

The secondary flow velocities in rectangular pipes were measured in experiments [6]. In three pipes with the aspect ratios of the cross-sectional rectangle $b: a=1: 1,2: 1$, and 3:1 maximum values turned out to be approximately the same, amounting to $1.5 \%$ of the velocity at the pipe center. Everywhere a secondary flow velocity maximum is reached near the wall. On the corner bisectors the velocity is not greater than $1 \%$. Within any corner the secondary flow takes the shape of a pair of vortices of opposite signs separated by the bisector. A small-sized vortex is located along the short side of the rectangle and the larger vortex lies along the long side. As distinct from [2], where the presence of several vortices along the long rectangle side was supposed, in all the cases studied only one vortex was observable; the motion intensity within it decreased with distance from the corner. The mean velocity distribution and the secondary flow streamlines in a rectangular pipe with the aspect ratio 4:1 obtained in numerical calculations [34] at $\mathrm{Re}=4410$ $\left(\operatorname{Re}_{\tau}=150\right)$ are presented in Fig. 6. Only the left halves of the pipe cross-section are shown. The velocity contour shapes are in qualitative agreement with Nikuradse's results [1] presented in Fig. 1. The distinctive feature of the velocity distribution is the characteristic convexities of the isolines toward the corners. The shape of the secondary flow streamlines is in agreement with the results [6] and argues against Prandtl's supposition [2] about the presence of several circulation cells along the long rectangle side (Fig. 2).

Turbulent flows in pipes of rectangular cross-section were considered in [35] $\left(\operatorname{Re}_{\tau} \approx 300, b / a=3.33\right)$, [36] (LES, $\operatorname{Re}_{\tau} \approx 150, b / a=1-4$ ), and in a series of studies by Vinuesa et al. [37-40] in the ranges $\operatorname{Re}_{\tau}=180-360$ and $b / a=1-14.4$. Variations in the secondary flow shape occurring with increase in the $b / a$ ratio and the effect of the side walls of the pipe on the flow in the central region were studied. The results of those studies were summarized in [40]. The secondary flow effect extends on large distances from the corner. The velocity profile in the section farthest from the corner acquires the asymptotic shape 
only for $b / a>10$. For the purpose of possible attenuation of the secondary flow Vidal et al. [41-43] investigated flow in rectangular tubes with rounded corners. Contrary to the expectations, the rounding of the corners did not lead to the secondary flow attenuation and in certain cases even favored its enhancement. The vortices are formed near the junctions between the roundednesses and straight regions. In the cases in which a straight lateral wall in a rectangular pipe was replaced by a semicircular wall, only one vortex each is formed near the junction between the semicircle and the long rectilinear walls rather than conventional four vortices (a vortex pair each near each angle).

In [9] turbulent flow in pipes of elliptical cross-section were calculated. The calculations were carried out in the Cartesian coordinate system. The no-slip boundary conditions on the curvilinear boundaries were satisfied using the method of virtual boundaries [44]. The flows in the pipes with ellipse semiaxis ratios $b / a=2 / 3$ and $1 / 2$ were studied for $\operatorname{Re}=6000$. It was found that in both cases the secondary flows take the form of two vortex pairs of opposite sign. The fluid flows from the pipe center toward the walls along the large semiaxes, spreads along the walls to both sides, and returns from the walls to the pipe center along the short semiaxes. In the flow core the greatest secondary flow velocity is less than $1 \%$ of the mean velocity, while near the walls it amounts to 1 and $1.4 \%$ in the wide and narrow pipes, respectively. Despite the so small intensity, the secondary flows have a considerable effect on the mean flow velocity distribution in the pipe cross-section, which is comparable with the effect of turbulent stresses. The effects of the secondary flows and turbulent stresses are opposite: the former tend to extend the velocity contours along the large ellipse semiaxes, whereas the turbulent stresses constrict them toward the pipe center. To more accurately reproduce the flow details in the wall region in $[45,46]$ the calculations of flows in elliptical pipes were carried out. In those studies the Navier-Stokes equations were solved in the elliptical cylindrical coordinate system in which the pipe wall coincides with a coordinate surface. The method of solving the Navier-Stokes equations in an arbitrary curvilinear coordinate system [47] was used. The inferences made in [9] were confirmed and refined and the terms of Eq. (1.1) for the longitudinal vorticity were assessed. The source term $S$ on the right side of Eq. (1.1) has noticeable nonzero values only in a narrow wall layer, where it can be represented similarly to Eq. (1.2) in the form of the sum of two terms, of which the first, $S_{1}$, is the second mixed derivative of the difference between the normal Reynolds stresses, while the second, $S_{2}$, is the difference of the second derivatives of the Reynolds shear stress. Elementary estimates show that $S_{1}$ and $S_{2}$ act as the longitudinal vorticity source and sink, respectively. The $S_{1}$ and $-S_{2}$ distributions in the pipe cross-section turn out to be surprisingly similar in shape, $\left|S_{2}\right|$ amounting to about $30 \%$ of $\left|S_{1}\right|$.

In the pipes of circular cross-section secondary flows do not occur due to the central symmetry of the flow. Obviously that in the pipes of annular cross-section secondary flows do not arise for the same reason. Secondary flows in the pipes with eccentric annular cross-section were studied experimentally in [48, 49]. According to measurements [48], the secondary flow takes the shape of pairwise, oppositely directed vortices, placed symmetrically relative to the line of symmetry in the cross-sectional plane. Circulation cells are mostly pressed against the inner cylinder. The fluid moves from the narrow gap toward the wide gap along the inner cylinder and returns backward, approximately midway between the cylinders. In the widest part of the gap the secondary flow velocity is directed from the inner to the outer cylinder. In [49] the secondary flow direction was opposite. Moreover, in the narrow part of the gap one more pair of vortices of smaller intensity was noticed. We note that the plausibility of both results is not high, which was admitted by their authors themselves. Moreover, the measurements in the two cited experiments were conducted for considerably differing geometric parameters and Reynolds numbers. In [50, 51] direct calculations of turbulent flow in eccentric annular pipes were carried out at two Reynolds numbers $\mathrm{Re}=4000$ and 8000 . The algorithm [47] was applied in the bicylindrical coordinate system, in which both pipe walls are coordinate surfaces. The secondary flow pattern thus obtained resembles the results of [48]: the fluid particles move along the inner cylinder from the narrow to the wide gap and return backward in the mid-cross-section. Nearer to the outer cylinder, from the side of the wide gap there is a less intense circulation cell with opposite rotation direction. Topologically equivalent secondary flow pattern with two vortices of opposite sign in each half of the cross-section was obtained numerically in [52]. However, as distinct from [50,51], the less intense vortex is located in the narrow rather than in the wide part of the gap. These differences in the secondary flow shapes can be attributed to a considerable difference in the geometries of the pipes considered in [50,51] and [52].

Secondary flows along external corners are studied in less detail. We can note only the experiments performed in the Melbourne University [53-55] and numerical calculations [56, 57]. In the experiments a flow along a ridge formed by two flat plates joined at right angle was studied. In the calculations flow in in a gap between two pipes of square cross-section nested within each other was considered; the pipe had 
(a)

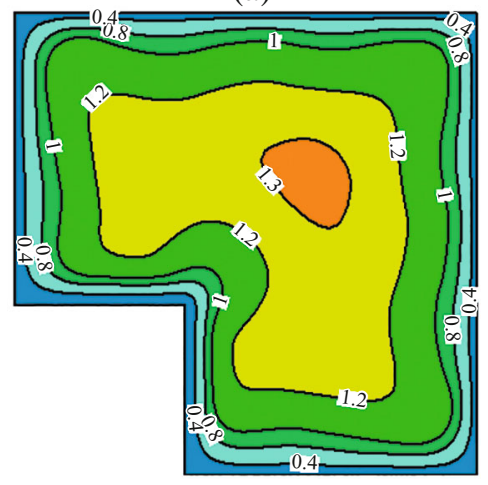

(b)

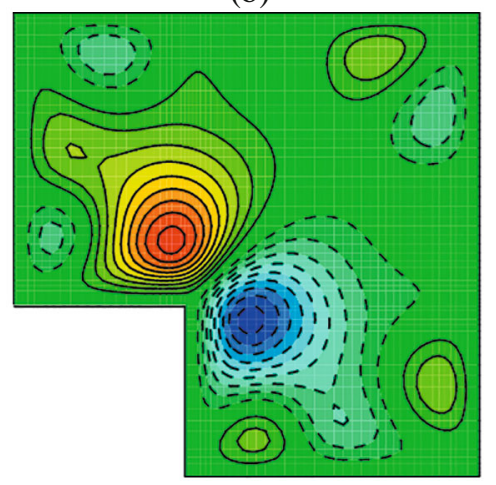

Fig. 7. Distribution of the mean velocity $U / U_{b}$ (a) and secondary flow streamlines (stream function contours $\left.\Psi h / U_{b}= \pm 0.0004 n, n=1-9\right)(\mathrm{b})$ in the cross-section of a pipe with an external angle at Re $=4000$ [58].

four internal and four external $90^{\circ}$ corners. Although the Reynolds numbers in the calculations and experiments were considerably different, the results concerning the secondary flow features in the vicinity of the external corner are in qualitative agreement. In Fig. 7 we have plotted that mean velocity distribution and the secondary flow streamlines in the pipe, whose cross-section has an external corner [58]. The secondary flow in the vicinity of the external corner takes the shape of a pair of vortices placed symmetrically relative to the corner bisector. The fluid motion in the secondary flow is directed from the corner toward the flow along the bisector and toward the corner along the walls, that is, it is opposite to the case of the internal corner. Correspondingly, the velocity contours have characteristic convexities directed from the corner toward the flow. In the vicinity of the external corner the secondary flow intensity amounts to $5.25 \%$ of $U_{b}$, which is considerably greater than the analogous intensity near the internal corners, where a maximum value is about 2\%. In [57] the distributions of the terms $S_{1}$ and $S_{2}$ and the expressions for the longitudinal vorticity production (1.2) were calculated. As in the vicinities of the internal angles, $S_{1}$ and $S_{2}$ turn out to be similar in value and opposite in sign. Their role in the vorticity generation changes in different parts of the vicinity of the corner.

Secondary flows in the channels with free surfaces have been actively studied in view of their obvious geophysical importance. The secondary flows are associated with the zones of fluid elevation and lowering that comprise the entire flow thickness. In the full-scale conditions the secondary flows have a considerable effect on gas and heat transfer and the self-aeration on the free surface. Thanks to the transport of bottom deposits by secondary flows, the bottom shape changes and the bottom roughness becomes inhomogeneous, with the formation of longitudinal ridges and depressions, which enhances the secondary flows and ultimately leads to the generation of a chain of longitudinal vortices with alternate direction of rotation in the channel width. For this reason, the greatest attention is given to an investigation of flows in channels with a rough bottom in the presence of inhomogeneities of different type in the bottom roughness distributions [59-64].

It is generally believed that at the initial stage of development the secondary flows arise in corner regions, where lateral walls are joined with the horizontal bottom. In accordance with the currently accepted point of view, the secondary corner flows in a smooth channel have a shape of two cells, lower and upper, with opposite directions of fluid rotation (Fig. 8) [60]. The motion in the upper cell is directed from the lateral wall along the free surface and it is more intense than the flow in the lower cell. With distance from the lateral wall the intensity of secondary corner flows is attenuated and vanishes completely at a distance of about $3 h$, where $h$ is the channel depth [65]. We note that the pattern of the secondary corner flow in an open channel described above is different from the secondary flow in a rectangular pipe. In the latter case two, rather than one, vortex pairs are observable near each lateral wall. A detailed experimental and numerical investigation of the region of junction between a free boundary and a solid wall [66] revealed the occurrence of a less intense "internal" secondary vortex, within which the motion is directed toward the corner along the free boundary. An investigation of the mechanisms of the momentum and energy transfer in this region was performed in [67]. 


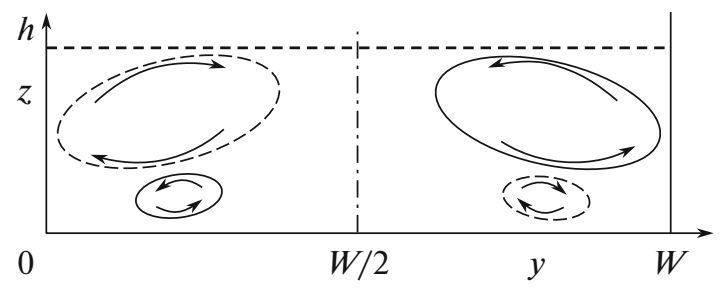

Fig. 8. Schematic of secondary flows in an open channel [60].

\section{PHYSICAL MECHANISM OF SECONDARY FLOW GENERATION IN NONCIRCULAR PIPES}

Despite the great many of experimental, theoretical, and numerical studies devoted to an investigation of Prandtl's secondary flows of the second kind, the question of the physical mechanism of their formation is still continued to be discussed in the literature. In many studies the reasoning is based on an analysis of the source terms (1.2) in equation (1.1) for the mean longitudinal vorticity. The relative importance of two terms, namely, that with the difference of the normal stresses $S_{1}$ and that with the shear stresses $S_{2}$ is being assessed. This subdivision of the source term was introduced in [16] for pipes of rectangular crosssection, when the coordinate axes are parallel to the pipe walls. This fact is frequently left out of account and a special physical meaning is ascribed to the terms $S_{1}$ and $S_{2}$ in analyzing flows in pipes with arbitrary cross-sections [21]. In the meantime, their values vary, when the coordinate system is rotated in the $(y, z)$ plane and, in particular, switch places, when the axes are rotated at an angle of $45^{\circ}$ [19]. In other words, if $v_{1}^{\prime}$ and $w_{1}^{\prime}$ are the fluctuations of the velocity components corresponding to the $y_{1}, z_{1}$, coordinate system rotated by an angle of $45^{\circ}$, then

$$
\frac{\partial^{2}}{\partial y_{1} \partial z_{1}}\left(\left\langle v_{1}^{\prime 2}\right\rangle-\left\langle w_{1}^{\prime 2}\right\rangle\right)=\left(\frac{\partial^{2}}{\partial z^{2}}-\frac{\partial^{2}}{\partial y^{2}}\right)\left\langle v^{\prime} w^{\prime}\right\rangle ; \quad\left(\frac{\partial^{2}}{\partial z_{1}^{2}}-\frac{\partial^{2}}{\partial y_{1}^{2}}\right)\left\langle v_{1}^{\prime} w_{1}^{\prime}\right\rangle=\frac{\partial^{2}}{\partial y \partial z}\left(\left\langle v^{\prime 2}\right\rangle-\left\langle w^{\prime 2}\right\rangle\right) .
$$

For this reason, the use of the source term in form (1.2) for describing the secondary flows in pipes with other-than-rectangular cross-sections seems weakly productive. The quantities $S_{1}$ and $S_{2}$ being nonzero individually can completely neutralize one another, as it is the case, for example, in circular pipes. Moreover, even in rectangular pipes the $S_{1}$ and $S_{2}$ distributions are similar in shape but their values are opposite in sign, so that they neutralize one another by $90 \%$ [26].

An alternative representation of the source term was proposed in [28]

$$
S=\left(\left\langle\omega_{x}^{\prime} \frac{\partial u^{\prime}}{\partial x}\right\rangle-\left\langle u^{\prime} \frac{\partial \omega_{x}^{\prime}}{\partial x}\right\rangle\right)+\left(\left\langle\omega_{y}^{\prime} \frac{\partial u^{\prime}}{\partial y}\right\rangle+\left\langle\omega_{z}^{\prime} \frac{\partial u^{\prime}}{\partial z}\right\rangle-\left\langle v^{\prime} \frac{\partial \omega_{x}^{\prime}}{\partial y}\right\rangle-\left\langle w^{\prime} \frac{\partial \omega_{x}^{\prime}}{\partial z}\right\rangle\right) \equiv S_{1}^{\prime}+S_{2}^{\prime} .
$$

In this writing each of the terms in the parentheses is invariant with respect to the choice of the coordinate system in the $(y, z)$ plane. In $x$-homogeneous flow we have $-\left\langle u^{\prime} \partial \omega_{x}^{\prime} / \partial x\right\rangle=\left\langle\omega_{x}^{\prime} \partial u^{\prime} / \partial x\right\rangle$, so that

$$
S_{1}^{\prime}=2\left\langle\omega_{x}^{\prime} \frac{\partial u^{\prime}}{\partial x}\right\rangle
$$

This quantity describes the effect of the longitudinal vorticity variation under the action of the pulsation-driven compression/extension of vortex tubes. It turns out that in the region of $\Omega_{x}$ concentration the production of the mean longitudinal vorticity in rectangular pipes is in a great measure controlled by the term $S_{1}^{\prime}$. This means, in particular, that the fluctuations of the longitudinal velocity and vorticity components occur consistently, so that the correlations of $\omega_{x}^{\prime}$ and $\partial u^{\prime} / \partial x$ are consistent with $\Omega_{x}$ in the value and the sign. In [28] an explanation of the reason for this consistence was proposed.

Starting from [27], attempts have been made to relate the secondary flows with the special features of turbulent large-scale structures (near-wall streaks and quasi-longitudinal vortices) and phenomena (sweepings and ejections) observed in the wall layers of turbulent flows (see [30, 39, 43, 68-70] and others). In our opinion, these observations only indicate the existence of certain features of turbulent structures in the regions of the secondary flow generation but do not reveal the mechanisms of this generation. 
(a)

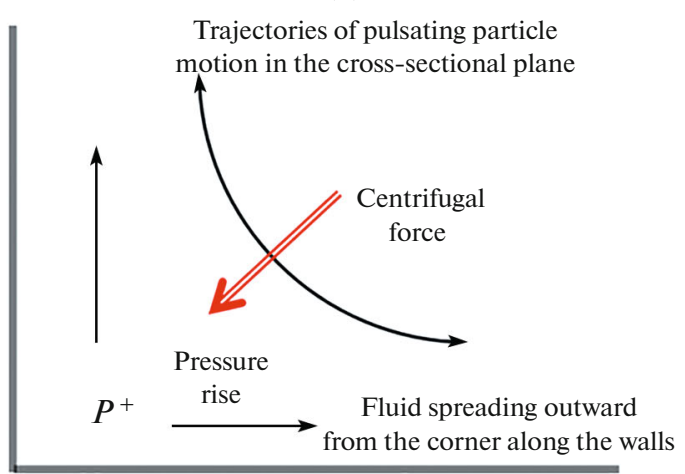

(b)

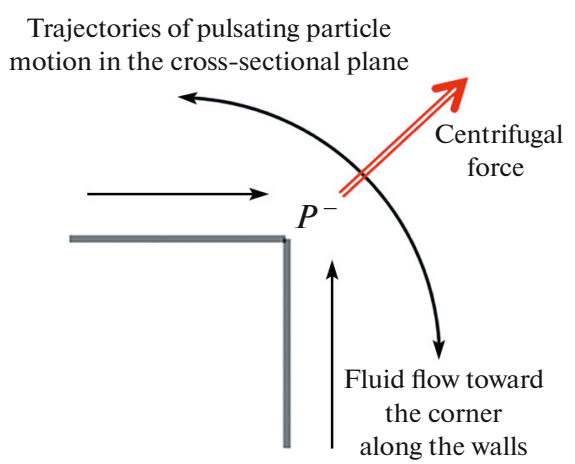

Fig. 9. Mechanism of secondary flow formation in the vicinities of internal (a) and external (b) corners.

This point of view is favored by the fact that the secondary flows can be observable in the absence of the main turbulent stream [71].

In [72] a theoretical analysis of the development of a turbulent boundary layer in a flow along a $90^{\circ}$ internal corner was made. The flow was considered at a considerable distance from the leading edge, where the boundary-layer approximation is valid. It is held that secondary flows are absent. In this case, the Reynolds equations give the relation between the mean pressure and the Reynolds stresses. Let the corner bisector in the cross-sectional plane intersect the outer edge of the boundary layer at point $A$, while points $B$ and $C$ are the point $A$ projections on the lateral walls. Under certain additional assumptions we obtain that the pressure $P(O)$ at the corner point $O$ is greater than the wall pressure at points $B$ and $C$. Hence it follows that under the pressure difference the fluid must move along the wall in both sides from the corner point and flow toward the corner along the bisector. Thus, the well-known secondary flow pattern within a corner is obtained. In [72] it was also assumed that in a developing boundary layer there arises a chain of secondary vortices, whose characteristic dimensions increase with boundary layer broadening. However, this model was not confirmed in experiments [73]. Moreover, it was found that at the spatial development stage the primary Reynolds stresses $\left\langle u^{\prime} v^{\prime}\right\rangle$ and $\left\langle u^{\prime} w^{\prime}\right\rangle$ make a more considerable contribution to the secondary flow formation than the Reynolds stresses associated with pulsations in the $(y, z)$ plane.

The reasoning of [72] was repeated with minimum corrections in [74] for the case of a steady flow in a pipe of square cross-section. The center of the pipe cross-section is taken for point $A$. As a result, it turns out that the pressure $P(O)$ at the corner vertex is greater than the pressures $P(B)$ and $P(C)$ at the midpoints of the lateral walls of the cross-section, whence it follows that the pressure gradient leads to the fluid spreading along the walls outward from the corner. We note that in both cited papers the conclusion that a local pressure maximum is reached at the corner point is made basing on the $P(O)>P(B)$ inequality. Theoretically, a situation is possible, when with distance from the corner the pressure first increases from the $P(O)$ value to a certain, an even greater value and only then starts to decrease down to $P(B)$. In this case the secondary flow direction near the corner must be opposite to the known direction. Moreover, the results $[72,74]$ pertain exceptionally to a $90^{\circ}$ corner and do not give idea of the behavior of secondary flows in other geometric configurations.

A variation of the mean pressure at the corner points of the cross-section can be explained basing on simple qualitative considerations described below with reference to the examples of flows along the internal and external $90^{\circ}$ corners. In the wall layer tangential fluctuations of the velocity are greater than the normal fluctuations, so that fluid particles move chiefly parallel to the walls, while in the corner region of the cross-section they move along arc-shaped paths joining the directions along the tangents to the neighboring boundaries (Fig. 9). The centrifugal force that arises in this case (inertia force) is directed toward the trajectory convexity. It causes a variation in the mean pressure in the corner and a pressure gradient acting radially in all directions. On the solid pipe walls the Reynolds stresses and their gradients are zero, so that the action of the pressure gradient along the walls can be offset only by the viscous friction forces. This necessitates the fluid motion along the walls from the greater to the smaller pressure. In the vicinity of the internal corner the centrifugal force is directed toward the corner, where a pressure rise occurs (Fig. 9a). On the contrary, in the vicinity of the external corner the centrifugal force is directed outward from the corner, which leads to rarefaction at the corner point (Fig. 9b). Correspondingly, within the 

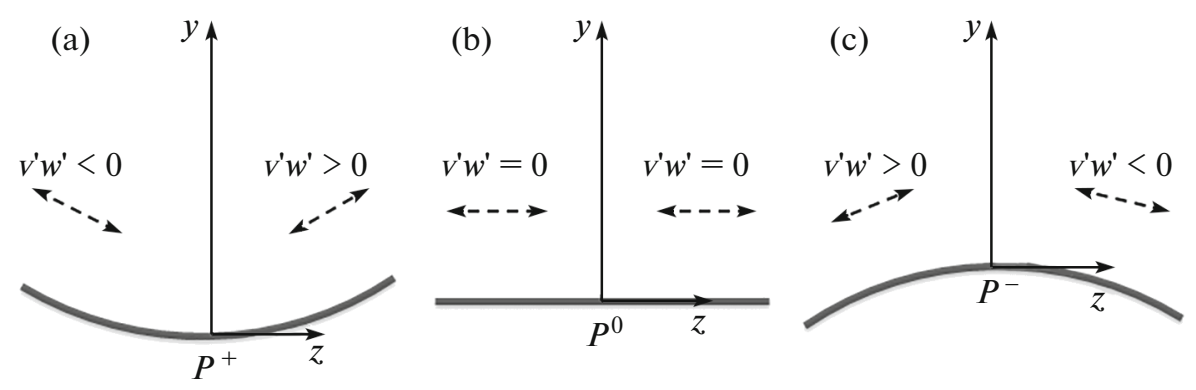

Fig. 10. Predominant directions of the velocity pulsations and products $v^{\prime} w^{\prime}$ in the near-wall region for the boundaries of three types: concave (a), plane (b), and convex (c). The designations $P^{+}, P^{-}$, and $P^{0}$ represent the positive, negative, and neutral effects of the second term on the right side of Eq. (3.1) on the wall pressure.

internal corners the fluid spreads along the walls outward from the corner, which is compensated by fluid inflow along the bisector, whereas in the vicinities of the external corners the fluid flows toward the corner along the walls and flows outward in the direction of the bisector.

\section{RELATION BETWEEN THE WALL PRESSURE AND THE BOUNDARY CURVATURE}

The prediction of secondary flows on the basis of an analysis of the right sides of Eq. (1.1) is not easy. In most cases, it is difficult to assess beforehand the cross-sectional Reynolds-stress distributions in pipes without invoking to numerical or laboratory experiments. The sole reliable apriori knowledge pertains to their behavior near solid smooth walls, where, by virtue of the no-slip conditions, all the components of the Reynolds stress tensor are zero, together with their gradients. For this reason, if a pressure gradient acts along the cross-section perimeter, then a secondary flow must necessarily arise, with the fluid flow direction along the wall from the greater to the smaller pressure. This flow compensates the pressure force by the viscous friction. The question about the relationship between the mean wall pressure and the boundary curvature was analyzed in detail in [75]. Here, we will present the main elements of that analysis.

The boundary curvature effect on the near-wall pressure can be estimated basing on the following simple considerations. We will consider three typical cases of the boundary shape, namely, concave, plane, and convex (Figs. 10a-10c). We will fix a certain point on the boundary and introduce a coordinate system $(y, z)$ with the axes aligned with the normal and tangent to the boundary at the chosen point. The Reynolds equation in the $y$ direction, when the secondary flow is neglected, gives the relation between the mean pressure gradient and the Reynolds stresses

$$
-\frac{\partial P}{\partial y}=\frac{\partial\left\langle v^{\prime 2}\right\rangle}{\partial y}+\frac{\partial\left\langle v^{\prime} w^{\prime}\right\rangle}{\partial z}
$$

Near the solid wall the first term on the right side of Eq. (3.1) is always positive and tends to increase the mean pressure on the wall. The sign of the second term is determined by that of the boundary curvature. The predominant pulsation direction in the wall region is parallel to the direction of the tangent at the nearest point of the boundary. For this reason, in the case of a concave wall (Fig. 10a) the Reynolds shear stress $\left\langle v^{\prime} w^{\prime}\right\rangle<0$ at $z<0$ and $\left\langle v^{\prime} w^{\prime}\right\rangle>0$ at $z>0$. Thus, the second term on the right side of Eq. (3.1) is also positive, which ensures an additional (compared with the first term effect) pressure increase at the boundary. This fact is noted by the designation $P^{+}$in Fig. 10a. The analogous reasoning for the vicinity of a convex boundary (Fig. 10c) leads to the conclusion that in this case $\partial\left\langle v^{\prime} w^{\prime}\right\rangle / \partial z<0$, that is, the Reynolds shear stress effect on the near-wall pressure is negative. Finally, in the plane region of the boundary it may be assumed that $\left\langle v^{\prime} w^{\prime}\right\rangle \approx 0$ on either side of the line $z=0$, so that the contribution of the second term on the right side of Eq. (3.1) is unimportant.

The qualitative estimates given above can be presented more formally. To do this, in [75] the notion of the pulsation trajectory was introduced; it is defined as a line in the cross-section, such that the direction of the tangent to it coincides at each point with the main direction of the Reynolds stress tensor corresponding to the greater principal stress. If $v_{\tau}^{\prime}$ and $v_{n}^{\prime}$ are the tangential and normal components of the 
velocity fluctuation components along the trajectory, then $\left.\left\langle v_{\tau}^{\prime 2}\right\rangle\right\rangle\left\langle v_{n}^{\prime 2}\right\rangle$ and $\left\langle v_{\tau}^{\prime} v_{n}^{\prime}\right\rangle=0$. It was proved that an any point on the trajectory

$$
-\frac{\partial P}{\partial n}=\frac{\partial\left\langle v_{n}^{\prime 2}\right\rangle}{\partial n}+\frac{\left\langle v_{\tau}^{\prime 2}\right\rangle-\left\langle v_{n}^{\prime 2}\right\rangle}{R} .
$$

Here, $\mathbf{n}$ is the principal normal and $R$ is the radius of curvature of the trajectory. The first term on the right side of Eq. (3.2) indicates that the pressure variation along the normal is opposite to the normal stress variation in the same direction and does not depend on the curvature of the pulsation trajectory. The second term is always positive, whence it follows that the fluctuating motion along a curved trajectory increases the pressure in the direction of the trajectory convexity. This increase is proportional to the trajectory curvature.

The curving of the pulsation trajectories takes place near curved walls, where $\left\langle v_{\tau}^{\prime 2}\right\rangle \sim d^{2}$ and $\left\langle v_{n}^{\prime 2}\right\rangle \sim d^{4}$, $d$ being the distance to the wall. As $d \rightarrow 0,\left\langle v_{\tau}^{\prime 2}\right\rangle \gg\left\langle v_{n}^{\prime 2}\right\rangle$ and it may be assumed that the pulsation trajectory curvature is similar in value with the wall curvature. It is reasonable to suggest that the pulsation trajectory curvature retains the sign even at a certain distance from the wall, so that the effect of the second term on the right side of Eq. (3.2) leads to a finite variation in the wall pressure. Thus, with variation in the wall curvature a proportional variation of the pressure might be expected in the neighboring regions of the pipe cross-section perimeter: with increase in the wall curvature the pressure increases on concave walls and decreases on convex walls. As noted above, the Reynolds stresses cannot balance out the pressure force action along the wall, so that a secondary flow must arise. In accordance with the above, the motion in the secondary flow must be directed from the greater toward the smaller curvature along concave walls and vice versa along the convex walls.

Obviously that the curving of the pulsation trajectories occurs also in corner regions. The family of pulsation trajectories in a pipe of square cross-section for $\mathrm{Re}_{\tau}=150$ [28] is presented in Fig. 11a. The radius of curvature of the pulsation trajectories is zero directly at the corner point and increases with distance from it. A noticeable curving of the trajectories can be observable in the region, where $y^{+}, z^{+}<50$. In the same region there exists a considerable anisotropy of normal stresses, which makes a contribution to the second term on the right side of Eq. (3.2). In Fig. 11b the pressure field is presented, together with the secondary flow streamlines. Precisely in the zone of curving of the pulsation trajectories an additional wall pressure rise is observable, as compared with the boundary regions, more distant from the corner. Beyond this region the pulsation trajectories are parallel to the walls and it is only the first term on the right side of Eq. (3.2) that contributes to the wall pressure rise. In the internal corners both terms on the right side of Eq. (3.2) are positive and their effects are added. In the vicinities of the external corners the pulsation trajectories are directed outward from the corner by their convexities, so that the second term on the right side of Eq. (3.2) tends to diminish the pressure in the corner. This leads to rarefaction (relative to the surrounding regions of the perimeter) in the corner region and a secondary flow directed along the walls toward the corner and along the bisector outward from the corner.

\section{PREDICTION AND EXPLANATION OF THE SECONDARY FLOW SHAPE IN NONCIRCULAR PIPES}

The arguments and estimates of the preceding section concerning the relation between the curvature of the pipe cross-section boundary and the mean pressure and the direction of the generated secondary flow can be formulated in the form of the following principle. The secondary flow along the pipe walls is directed from the points of local pressure maximum to those of local pressure minimum. The consequence of this principle is the fluid inflow from the external flow toward the points of local pressure maxima on the walls and, contrariwise, the fluid return from the walls into the external flow from the points of local pressure minimum. In the vicinity of any extremum the streamlines take the form presented in Fig. 12. At the points of local extrema of the boundary curvature the pressure extrema of the same type are reached on concave walls and of the opposite type on convex walls.

This principle can be used for approximately estimating and predicting the shape of the secondary flows occurring in one or another version of the pipe cross-section. For this purpose, an attempt should be made to determine the points of local pressure extrema on the pipe cross-section boundary basing on an analysis of the boundary curvature, symmetry conditions, or some other considerations. Two extrema of the same type cannot be next to one another on an interval of a boundary but they must necessarily be separated by at least one extremum of the opposite type. 


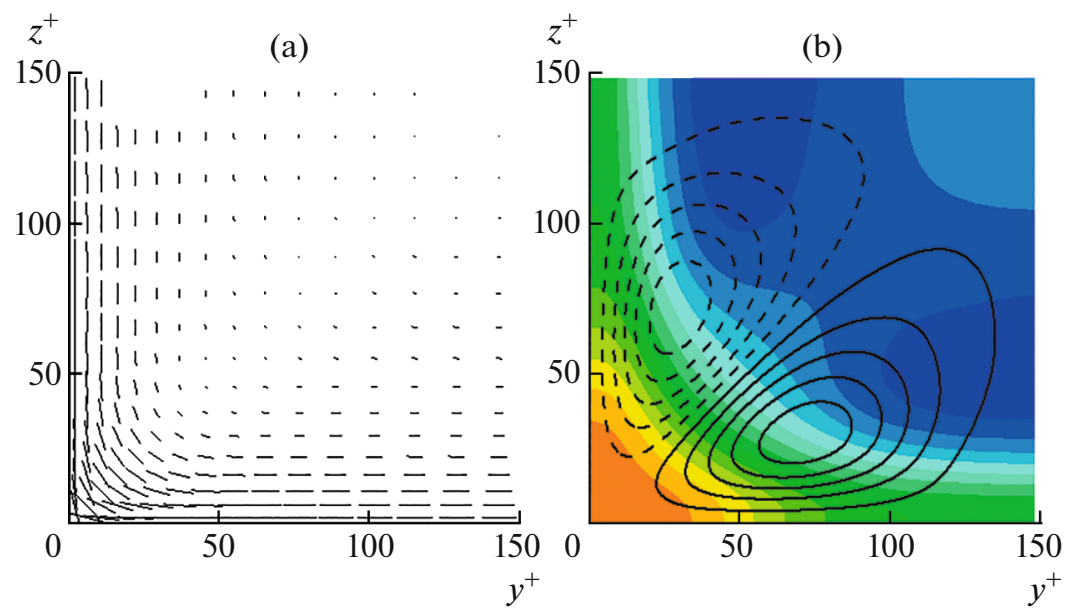

Fig. 11. Directions of the tangents to pulsation trajectories in a square pipe (a) and mean pressure field and secondary flow streamlines (b) at $\operatorname{Re}_{\tau}=150$. The vector lengths in panel (a) are proportional to the anisotropy degree of the normal stresses $\left(\left\langle v_{\tau}^{\prime 2}\right\rangle-\left\langle v_{n}^{\prime 2}\right\rangle\right) /\left(\left\langle v_{\tau}^{\prime 2}\right\rangle+\left\langle v_{n}^{\prime 2}\right\rangle\right)$. A quarter pipe cross-section is presented.
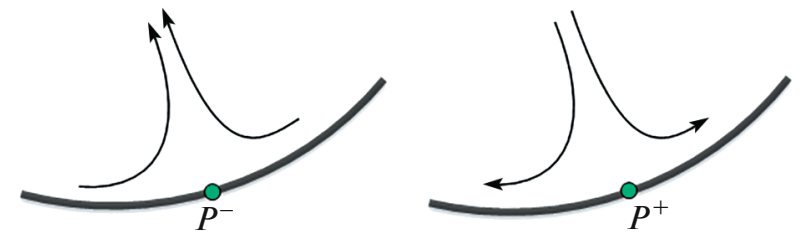

Fig. 12. Secondary flow streamline shapes in the vicinities of the points of local minimum $P^{-}$and maximum $P^{+}$of the mean wall pressure.
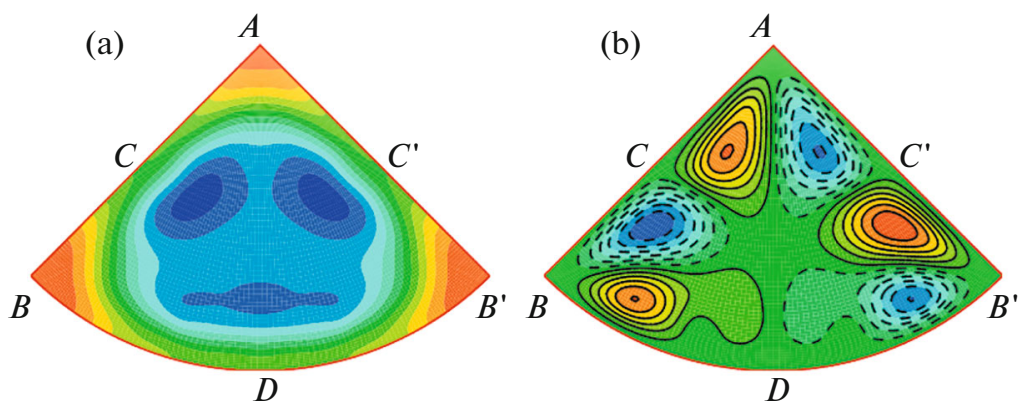

Fig. 13. Mean pressure distribution (a) and secondary flow streamlines (b) in a pipe with the cross-section in the shape of a circular sector with the vertex angle of $90^{\circ}$ [76].

We will try to apply the receipt presented above to certain particular cases. In Figs. 13 and 14 we have presented the pressure distributions and the secondary flow streamlines in the pipes with cross-sections in the shape of a circular sector with the vertex angles $90^{\circ}$ and $270^{\circ}$ obtained in the DNS calculations at $\operatorname{Re}=U_{b} D_{h} / \nu \approx 2800$ [76]. Here, $U_{b}$ is the mean velocity and $D_{h}$ is the hydraulic diameter.

An apriori analysis of the pipe cross-section with the vertex angle $90^{\circ}$ (Fig. 13) allows one to make the following conclusions. For the reasons described above, in three internal corners $A, B$, and $B^{\prime}$ the pressure is greater than on the surrounding intervals of the perimeter. On the rectilinear intervals $A B$ and $A B^{\prime}$ there must be points of local pressure minimum; we will denote them as $C$ and $C^{\prime}$. Any variations in the boundary curvature or any other reasons indicating the presence of other extrema are absent from these intervals. 

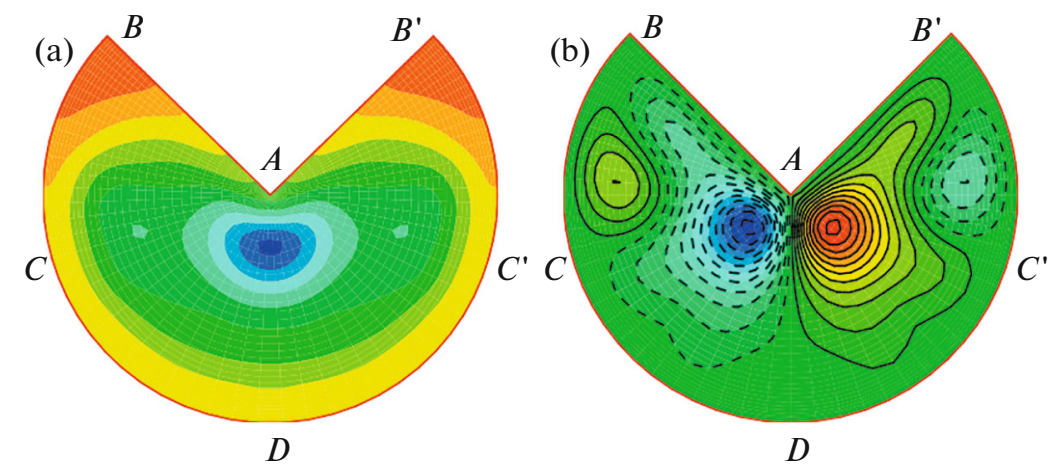

Fig. 14. Mean pressure distribution (a) and secondary flow streamlines (b) in a pipe with the cross-section in the shape of a circular sector with the vertex angle of $270^{\circ}[76]$.

At the midpoint $D$ of arc $B B^{\prime}$ an extremum must exist by virtue of symmetry. Since this point lies between two local maxima, this is apparently a local minimum. There remains to connect the points of pressure extremum in accordance with the rule shown in Fig. 12 without mutual intersections of the lines within the pipe in order to obtain an approximate streamline pattern in the secondary flow. In this case, the supposed secondary flow is qualitatively equivalent to the flow in the pipe of triangular cross-section [2] shown in Fig. 2. The fluid moves from the central region of the pipe toward the points of local pressure maxima located at the vertices of the three corners $B, B^{\prime}$, and $A$. The fluid flows out of the corners toward points $C, C^{\prime}$, and $D$ along the walls. From these points the fluid returns to the pipe center. The DNS results confirm generally the prognosis made concerning both the pressure distribution along the crosssection perimeter (Fig. 13a) and the shape the secondary flow streamlines (Fig. 13b). Small deviations from the predicted shapes of the secondary flows that take place in the bottom of Fig. 13b cannot be obtained on the basis of a comparatively coarse analysis.

We will now turn to the pipe with the vertex angle of $270^{\circ}$ (Fig. 14). We suppose the presence of pressure extrema at the corner points, namely, local maxima in the internal corners $B$ and $B^{\prime}$ and a local minimum in the external corner $A$. We note that in this case the pressure behavior is considered only along the perimeter. The local minimum at point $A$ means that in the surrounding regions of the boundary the pressure is greater but this does not mean a necessary increase in the pressure at the surrounding points within the pipe. At the midpoint $D$ of $\operatorname{arc} B B^{\prime}$ a local extremum must take place by virtue of symmetry. In this case, the type of this extremum is not obvious. The pressure behavior at this point is in a lesser degree determined by the distant corners $B$ and $B^{\prime}$ than by the corner $A$. In the vicinity of point $A$ the trajectory convexities are directed toward point $D$, which can manifest itself as a certain pressure rise in the vicinity of this point, compared with the surrounding intervals of arc $B B^{\prime}$. Thus, the presence of a local pressure maximum may be supposed at point $D$, together with the appearance of local minima at certain intermediate points $C$ and $C^{\prime \prime}$ on the arcs $B D$ and $B^{\prime} D$. In this case, the secondary flow along the boundary is directed from the corners $B$ and $B^{\prime}$ toward the corner $A$ and the points $C$ and $C^{\prime}$ and also from point $D$ toward the points $C$ and $C^{\prime}$. The fluid moves from the local minimum points $C, C$, and $A$ inward the pipe and then in the direction of points $B, B^{\prime}$, and $D$, respectively.

The pressure distribution along the perimeter described above and the ensuing secondary flow topology is confirmed by the results of numerical calculations presented in Fig. 14. We note that it was obtained on the basis of only one supposition that the pressure at point $D$ has a local maximum. An alternative supposition about a local pressure minimum at this point gives another pattern. In this case, the secondary flow along the $\operatorname{arcs} B D$ and $B^{\prime} D$ is directed toward point $D$ and from this point inward the pipe, where it meets the secondary flow from point $A$, whereupon both streams flow toward the corners $B$ and $B^{\prime}$. Although in this case this pattern is not confirmed by the DNS results, it can be realized for other Reynolds numbers or the values of the angle at vertex $A$.

What calls attention to itself in Fig. 14a is the characteristic pressure distribution along the radius $A D$ having a minimum at a certain intermediate point rather than at point $A$. The explanation of this fact is that the normal velocity fluctuations in the vicinity of the singular point $A$ are quite considerable, so that the negative first term on the right side of Eq. (3.2) overweighs the positive value of the second term related with the effect of the pulsation trajectory curvature. We note that the principal normal along the 


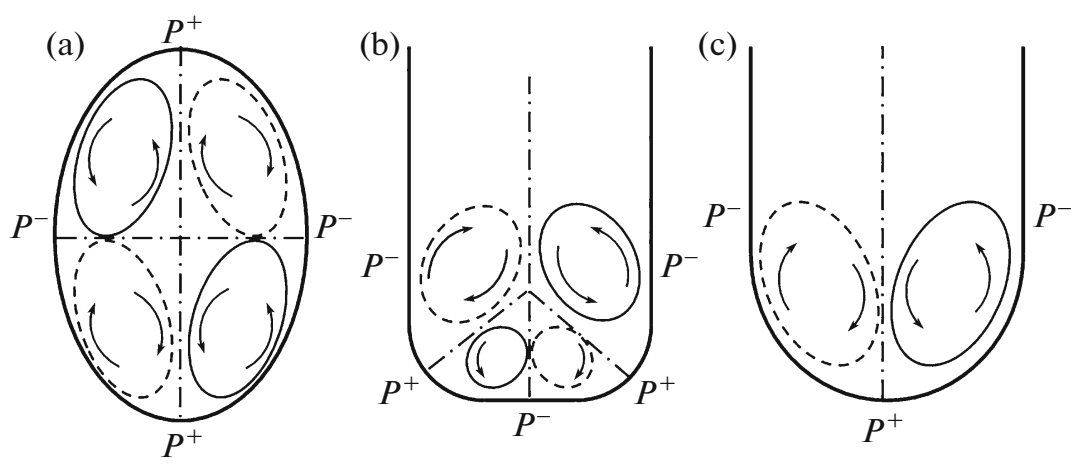

Fig. 15. Schematic secondary flow pattern in an elliptical pipe $[9,45,46](a)$, rectangular pipe with rounded corners [41] (b), and a pipe with a semicircular wall [41, 43] (c). The dashed and solid curves correspond to clockwise and counterclockwise rotation, respectively. The flow direction in secondary flows is additionally marked by arrows. The elevated and lowered pressure regions are denoted as $P^{+}$and $P^{-}$, respectively.

radius $A D$ oriented toward the concavity of the pulsation trajectory is in this case directed toward the corner point from the central region of the pipe and not in the opposite direction, as it is the case in internal corners.

We will consider some more cases known from the literature.

(i) Elliptical pipe [9, 45, 46], Fig. 15a. A maximum wall curvature is reached at the ends of the greater diameter; because of this, here there occurs a relative pressure rise $P^{+}$. Contrariwise, a minimum wall curvature and the lowered pressure $P^{-}$correspond to the ends of the smaller diameter. Thus, on each quarterperimeter the fluid flow along the wall is directed from the point of maximum pressure to that of minimum pressure, that is, from the ends of the greater diameter to those of the smaller diameter. The fluid is concentrated toward the center of the ellipse along the small semiaxes and spreads backward to the walls along the large semiaxes.

(ii) Rectangular pipe with rounded corners [41], Fig. 15b. In the corner regions, where the boundary curvature is maximum, the pressure $P^{+}$is elevated. Till the corner roundness radius is smaller than the channel half-width, the lower wall has a rectilinear region with a lowered pressure $P^{-}$. Thus, the secondary flow pattern coincides qualitatively with the case of unrounded corners: the fluid moves from the main stream to the points of elevated pressure in the corner region of the pipe and spreads from there along the walls to both sides, toward the lowered pressure zones.

(iii) Pipe with a semicircular wall [41, 43], Fig. 15c. When the plane region vanishes from the lower wall, the lowered pressure zone vanishes together with it. Correspondingly, the region of the secondary flow along the wall toward the vertical line of symmetry also vanishes and the four-vortex secondary flow pattern is replaced by a two-vortex pattern.

(iv) Annular eccentric pipe [50, 51], Fig. 16. A prediction of the secondary flow shape in an eccentric annular pipe is not so obvious. As for the pressure distribution over the pipe walls, it can be apriori affirmed with a great extent of assurance only that on the concave surface of the outer cylinder the pressure is greater than at the closest point on the convex surface of the inner cylinder. The pressure distributions along the perimeters of the inner and outer cylinders obtained in DNS calculations [51] are presented in Fig. 16b. The locations of local pressure maxima at all the boundaries are marked in Fig. 16a. The fluid flow direction along the walls is consistent with the pressure distribution. We note that the range of pressure variation along the innerl cylinder wall is twice as large as the analogous variation along the outer cylinder. In view of the fact that the inner perimeter length is half as large as that of the outer cylinder, the characteristic pressure gradient along the inner wall is by a factor of four greater than that along the outer wall. This exhibits itself as a considerably greater secondary flow intensity along the surface of the inner boundary on the interval $0.1<s<0.65$, where the fluid flows from the narrow to the wide gap. A weak motion along the outer cylinder occurs in the same direction. In the widest part of the gap, $s<0.2$, a pressure variation along the outer boundary stops and the secondary flow also vanishes. In the narrow part of the gap, $s>0.7$, the pressure decreases with increase in $s$ on both boundaries. In this part of the pipe the fluid must move toward the narrow gap. This is clearly visible on the outer cylinder in Fig. 16a, while on the inner cylinder this manifests itself in the form of detachment of the nearest-to-the-wall streamline 
(a)

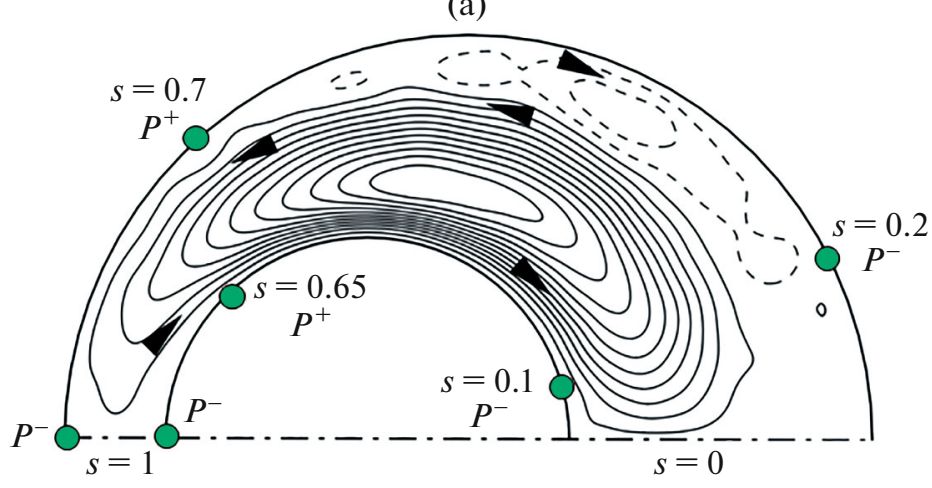

(b)

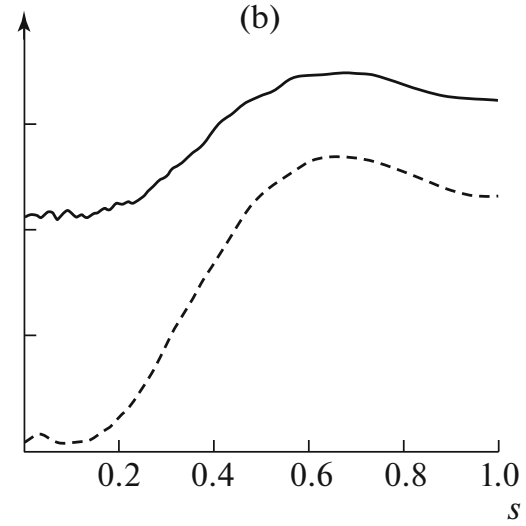

Fig. 16. Flow in an annular eccentric pipe [51]. The upper half of the cross-section is shown. Secondary flow streamlines (a) and mean pressure distributions along the perimeters of the inner (broken curve) and outer (solid curve) cylinders (b). The arrows indicate the motion direction in the secondary flow. The coordinate $s=0$ corresponds to the wide part of the gap and $s=1$ relates to the narrow part. The points on the cylinder walls indicate the locations of local extrema of the pressure $P^{+}$and $P^{-}$.

from the cylinder surface. Probably, nearer to the wall in this location there is a weak oppositely-directed motion, which is not shown in the figure.

\section{FLOWS IN CHANELS WITH FREE BOUNDARIES}

The mechanism of the secondary flow formation described in the previous sections is based on the assumption of the absence of Reynolds stresses from solid pipe boundaries. Under these conditions, the pressure variation along the boundary must be necessarily accompanied by a fluid flow from the greater toward the smaller pressure. The conditions of the secondary flow generation in channels with free boundaries are different from the case of a channel with solid walls in that on a free boundary there are tangential pulsations of the velocity, which are absent from a solid wall. Thus, the flow direction is governed by the competition between the pressure forces and the Reynolds stresses. An analysis of the conditions of the secondary flow generation in corner regions formed by solid and free boundaries was carried out in [75].

A flow in a rectangular channel with three solid walls and one (upper) plane wall free from tangential stresses was considered. Due to the reasons presented above, an elevated pressure zone must occur in each of the four corners of the cross-section, together with a pressure gradient expelling the fluid along the channel walls. Near the solid walls, where the Reynolds stresses, which could counteract the pressure force, are absent, a fluid motion outward from the corner must necessarily appear. However, the flow direction on the free surface can be, generally speaking, different, being determined by the competition between the pressure gradients and the Reynolds stresses. If the winner in this competition is the pressure force, then a secondary flow arises; it is analogous to the flow within a corner with solid walls: the fluid spreads along the walls in both directions, outward from the corner. In the case in which the pressure force cedes, the fluid in the secondary flow moves along the free surface toward the corner and is carried away downward along the solid wall, that is, a single-vortex secondary flow pattern appears in the upper corner. In any of the two possible scenarios in the vicinities of the upper corners the fluid must move downward along the solid lateral wall, which contradicts the secondary flow pattern, according to which the fluid flows toward the upper corner along the lateral wall and is carried away toward the channel center along the free boundary (Fig. 8) [60].

For studying the details of the secondary flow in the region of the junction between the free surface and a solid wall in [75] direct calculations of a turbulent flow in a rectangular channel with a 4:1 cross-section and a free boundary were carried out. The secondary flow streamlines are presented in Fig. 17a, together with the mean pressure field in the channel cross-section. The left of two symmetric channel halves is shown. As it was predicted, each corner of the cross-section, including the upper ones, contains an elevated pressure zone. On the free surface, near the wall the Reynolds stress action is directed toward the wall and is superior to the pressure gradient action; because of this, in this region the fluid flows toward the corner. Here a single upper corner vortex is formed, that is, of the two above-mentioned scenarios it 


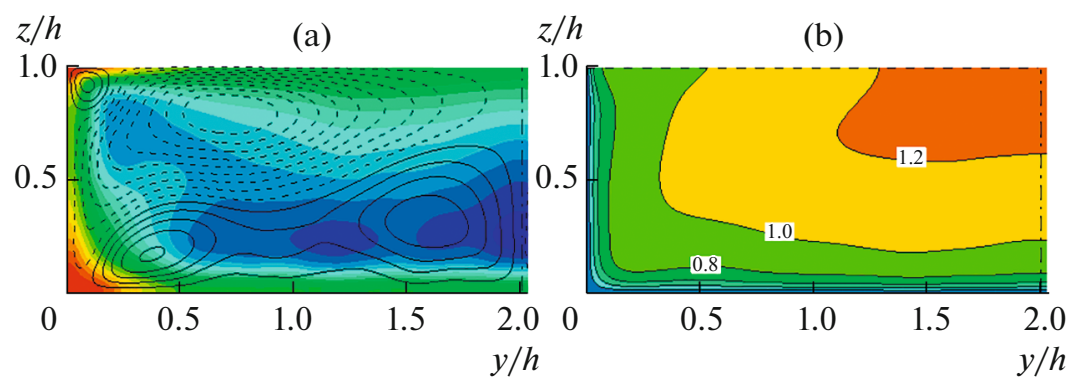

Fig. 17. Pressure field and secondary flow streamlines in the cross-section of a channel with a free upper boundary (a) and contours of the mean longitudinal velocity $U / U_{b}(\mathrm{~b})$.

is the second scenario is realized. It is in agreement with the results [66, 67] on the formation of an "internal vortex" in this region. In all other respects, the secondary flow pattern is in agreement with the conventional idea: the greater part of the channel cross-section is occupied by two intense vortices generated in the lower corners and penetrating inward the flow at a large distance. The secondary flow intensity turns out to be considerably greater than in a rectangular pipe with solid walls. It amounts to $2.5 \%$ in the vicinity of the solid walls and to $5 \%$ of $U_{b}$ on the free surface. The so large secondary flow velocity can be attributed to the fact that outside the wall region both the pressure gradient and the Reynolds stress force act in the same direction, outward from the corner. In the absence of wall friction here there arises an intense secondary flow which is transferred into other channel regions.

The contours of the mean longitudinal velocity $U$ plotted in Fig. $17 \mathrm{~b}$ have some distinctive features exhibiting the action of secondary flows. In the lower and upper corners these are the characteristic convexities toward the corners. The main singularity inherent in flows in open channels is a velocity increase beneath the free surface in the central region of the channel. This effect can be attributable to the surface transfer of the fluid having a low momentum from the near-boundary zones toward the channel center produced by the secondary flow.

An investigation of the flow in a channel with a free boundary [75] revealed some interesting properties of secondary flows. In particular, basing on the principle of secondary flow formation formulated above the possibility of the generation of a secondary flow in the form of a solitary vortex was predicted; this vortex arises in the region of the junction between the solid wall and the free surface in the cross-sectional plane $[66,67]$. The generation of the secondary vortex in this place is due to the presence of a wall with the no-slip condition on it, while the fact that only one vortex, rather than two, is formed is the distinctive feature of the interaction between a solid wall and a free boundary. It seems interesting to rise a question about the possibility and features of secondary flow formation in the corners, where two free boundaries are joined and a solid wall as an important element of the secondary flow formation mechanism is absent. A channel with two free boundaries is hardly realizable in experiments but it can be easily implemented in numerical simulations. In [75] direct calculations of turbulent flows in a channel of square cross-section were performed for the case, when two adjoining walls are solid, while the other two are free. In the calculations with several Reynolds numbers from $\mathrm{Re}=2000$ to $\mathrm{Re}=16000$ it was found that in the corner region near the junction between the two free boundaries the Reynolds stress forces turn out to be potential, accurate to computational errors. Thus, these forces are neutralized by the pressure gradient and do not produce a secondary flow. The secondary flow is conserved here only due to convection from distant regions.

\section{SECONDARY FLOWS NEAR ROUGH SURFACES}

The secondary flows of the second kind can be caused by the spanwise inhomogeneity of a rough surface in a flow. Under these conditions the secondary flow formation is of great importance, since in most of engineering applications and natural ecosystems turbulent flows occur precisely along rough surfaces, while smooth surfaces are rather rare. This phenomenon is particularly topical under the conditions of spatially inhomogeneous roughness; a well-known example is furnished by flows along the blades of turbomachinery [77]. An experimental investigation of turbulent flow over a damaged turbine blade with nonuniform surface roughness $[78,79]$ showed clearly the formation of secondary flows. Another important manifestation of secondary flows of this type is observable in river flows, where the spanwise transport of bottom sediments can produce and maintain changes in the bottom surface in a river bed [80]. 


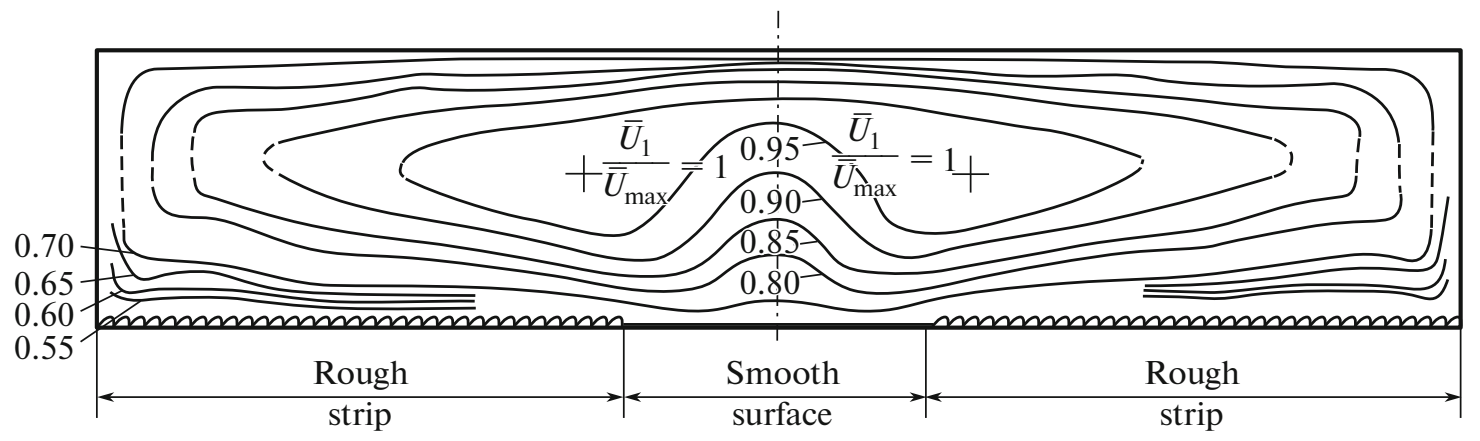

Fig. 18. Curving of the mean velocity contours in a rectangular pipe with an inhomogeneously rough wall [15].

The secondary flow formation over an inhomogeneously rough wall was first shown в [14, 15]. The roughness streaks aligned with the flow lead to the formation of an upward current over a smooth region of the wall and downward currents above the rough regions (Fig. 18). To explain this phenomenon in [14] the local production of the turbulent kinetic energy was compared with viscous dissipation; an imbalance between these quantities determines the motion topology and direction in the secondary flow. It was concluded that the fluid is transferred from less turbulent regions into the regions, where the turbulent kinetic energy production is superior to viscous dissipation, and vice versa. Basing on this assumption, in many subsequent studies the secondary flow topology and direction are associated with the turbulent kinetic energy distribution or the wall friction value, which are used for localizing the regions with greater/smaller turbulent activity.

As noted in [81], the term "roughness" is used sometimes by different research groups in different meanings. Two different types of the wall structuring can be meant under this term, namely, small-scale (about $10 \%$ of the boundary layer thickness) smooth structures, homogeneous in the flow direction and produced exceptionally by transverse variations in the wall height (for example, longitudinal ridges of different shapes on a smooth wall) and irregular rough surfaces with multidirectional variations in the surface height (for example, roughness streaks). The governing difference between these two types of surfaces is the absence of the pressure drag for the surfaces of the first kind (called sometimes "streamwise roughness") due to their homogeneity in the flow direction. In this configuration, all surface stresses are produced by viscous forces. Contrariwise, for the surfaces of the second kind with irregular roughness the pressure drag is predominant. This difference is also important for the mechanism of secondary flow formation. In the presence of a longitudinally-homogeneous structured surface the secondary flow formation can be attributed to the mechanisms described above for the internal and external corners in pipes. In the case of rough surfaces with streamwise inhomogeneities additional effects associated with the pressure drag should be taken into account. Despite a vast variety of different configurations of the transverse wall inhomogeneity, in recent decades two main configurations have been well studied. These are rough regions of walls arisen as a result of variations in the shear stress on the wall and transverse variations in the local wall elevation. In [80] it was proposed to subdivide the roughnesses into two types. When the effect of the shear stress variation is predominant, the roughness is called the strip-type roughness, while in the case of the wall elevation effect predominance it is the ridge-type roughness. The formation of secondary flows in an open channel was studied experimentally by means of placing rough surfaces of the both types. It was shown that the roughnesses generate large-scale secondary flows under the conditions, when the transverse inhomogeneity interval is comparable with the boundary layer thickness (Fig. 19). In this case, the observed upward and downward currents above the strip-type roughness occurring above both smooth and rough surfaces, whereas in the case of the ridge-type roughness the upward and downward currents were observable above elevated and lowered wall surfaces, respectively.

Characteristic of the strip-type roughness is an alternate spanwise variation in the shear stress on the wall, while variations in the wall height are absent or negligibly small. In $[82,83]$ numerical (DNS and LES) investigations of the strip-type roughness were carried out for the channel with strips of "small" and "large" roughness, which were realized by means of varying the boundary conditions on the wall ensuring a greater or smaller local friction. Both groups report on a secondary flow analogous to that observed in $[14,15]$, that is, upward above the region with the smaller friction and downward above the region with the greater friction. The secondary vortex structure thus obtained is consistent with experimental observations of flows over damaged turbine blades $[78,79]$. In these studies, strips with low and high flow velocities were found out, which can be related with the presence of large-scale secondary flows [68, 84]. As 


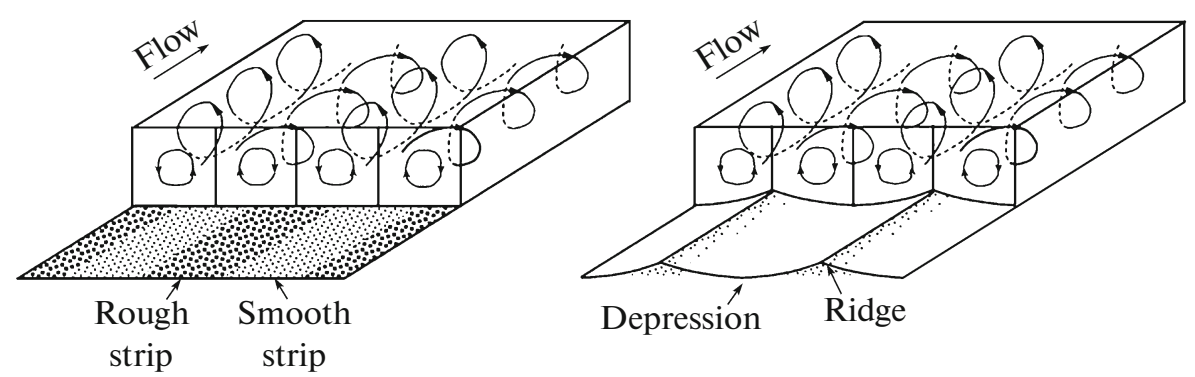

Fig. 19. Secondary flow over strip-type (left) and ridge-type (right) roughnesses [80].

shown in [82], the arrangement of the regions with opposite motion direction (upward or downward) in a secondary flow can change depending on the rough strip width. In [79, 85] a similar configuration was studied numerically in the case of a plane channel with alternating longitudinal strips and the slip and noslip conditions imposed on the walls. The authors report on an interchange of the secondary flow topology and direction with variation in the width of the region containing the slip.

Secondary flows over inhomogeneous surfaces made of spanwise alternating carton and sand-paper strips were studied experimentally in [86]. The results obtained can be summarized, follows: when the strip width is smaller than the boundary layer thickness, the secondary vortex dimensions are limited by this width (including those directed outward from the wall). If the strip width is of the order of the boundary layer thickness, then the secondary motion fills the entire boundary layer and has the greatest intensity. For the strips wider than the boundary layer thickness the secondary motion remains localized on the boundary between the rough and smooth strips and retains dimensions comparable with the boundary layer thickness.

The ridge-type roughness was also studied in detail by different research groups. Generally, it is observed that the length of an interval between elevated elements has an effect on the secondary flow topology and intensity, similarly to the strip-type roughness. Despite their small intensity, the secondary flows over the ridge-type roughness change considerably the main stream properties, which leads, in particular, to a considerable increase in friction and heat transfer [87]. In [88] a numerical investigation of the secondary flow over triangular streamwise ridges is presented. The authors relate the secondary flow formation with local spanwise redirecting of turbulent pulsations in the vicinity of the upper corner of the ridge outward from the wall, that is, with the appearance of nonzero correlation $\left\langle v^{\prime} w^{\prime}\right\rangle$. In [70, 89] turbulent flow over the seriss of blocks of Lego tinker toys was studied both experimentally and numerically. Despite an almost tenfold difference in the $\mathrm{Re}_{\tau}$ value, similar results were obtained in the calculations and experiment: the secondary flow consists of instantaneous large-scale vortices with primarily positive and negative vorticities to the right and left from an elevated element, respectively. The analogous experiments were performed in $[90,91]$ for different ridge geometries. Both research groups observed an upward motion over elevated elements. The results of a numerical investigation of a developing turbulent boundary layer over longitudinal rectangular ridges are presented in [92]. The authors relate a specific direction of secondary flows with a strong local imbalance of the turbulent kinetic energy due to large turbulent transport occurring above the ridge-type roughnesses, which was not observable in the case of strip-type roughness. The rotation direction in the secondary flows was related with an active transport of the turbulent kinetic energy into the region above the ridge (which is absent in the case of strip-type roughness), which, in author's opinion, is due to an upward deflection of transverse turbulent pulsations on the lateral walls of a roughness element. As shown in [87], the ridge geometry and height can have a considerable effect on the intensity of the generated secondary motion: sharp-cornered triangular ridges with a height of $16 \%$ of the channel half-height lead in this case to the secondary flow intensity amounting to $6 \%$ of the mean velocity, which is considerably higher than the secondary flow intensity usually observed in pipe corners or in the case of the strip-type roughness.

The subdivision of the inhomogeneous wall roughness into two types (strip and ridge types) makes easier the physical understanding of the phenomenon separating the effects of shear stress variations on a wall from the effects associated with wall elevations. However, it should be noted that a clear division between these two categories is impossible for actual roughnesses, since the presence of roughness is, itself, always accompanied by a variation in the roughness height. Thus, for example, in [93] a secondary flow in a turbulent plane channel, over a rough surface consisting of streamwise ranges of pyramids was investigated 
numerically for different spacings between the ranges. An effect of the change in the secondary flow direction was revealed with increase in the interval between the roughness ranges higher than the channel height. For smaller intervals an upward motion occurs above roughness elements, which is consistent with the secondary flows over the ridge-type roughnesses, while at large spacings a downward motion arises over the elements. Recently, in [94] it was shown that the rotation direction in the secondary flows above a wall with a roughness strip depends on the relative height of the mean level of the roughness strip (elevated or lowered). In that study three different levels of the mean height of the roughness strip were considered. They corresponded to elevated (caused by deposits), submerged (owing to erosion or corrosion), and intermediate roughness heights. Depending on this parameter, the secondary flow direction corresponded to either the strip-type or the ridge-type roughness.

\section{RANS-MODELING OF SECONDARY FLOWS}

The majority of numerical investigations of Prandtl's secondary flows of the second kind were performed using direct numerical simulation (DNS) or large eddy simulation (LES). The secondary flows become visible in calculations only as a result of the averaging of the velocity field over a considerable time interval. These investigations are computationally expensive even at low Reynolds numbers, since in the presence of only one homogeneous spatial direction a reliable averaging can be obtained when the flow is calculated during a considerable time. In predicting secondary flows the application of more cost-saving approaches based on the solution of the Reynolds equations (RANS-modeling) using semiempirical closure models turns out to be nontrivial matter owing to the absence of universal models. Moreover, the secondary flow generation is, in its essence, a result of the Reynolds stress anisotropy, which cannot be captured by the widely used classical models based on the assumption of the linear turbulent viscosity [95, 96]. Therefore, in describing secondary flows it is necessary to use specialized sophisticated turbulence models. In turn, this complexity puts bounds to the application and adoption of these models for solving engineering problems. The calculations become more expensive, while an extended numerical rigidity due to the nonlinearity reduces the model reliability leading to deterioration of convergence [97].

As can be seen from Eqs. (1.1) and (1.2), the secondary motion is generated due to the appearance of a difference between the Reynolds normal stress gradients $\left\langle v^{2}\right\rangle-\left\langle w^{\prime 2}\right\rangle$ and the transverse shear stress gradients $\left\langle v^{\prime} w^{\prime}\right\rangle$ [7]. Therefore, the capability of a turbulence model to predict Prandtl's secondary flows of the second kind is directly related with its capability of capturing the Reynolds stress anisotropy. The entire class of the closure models based on the linear Boussinesq relation supposes that the Reynolds stresses are determined by local mean strain rates

$$
-\left\langle u_{i}^{\prime} u_{j}^{\prime}\right\rangle=2 v_{t} S_{i j}-\frac{2}{3} k \delta_{i j}
$$

where $v_{t}$ is vortex viscosity, $k$ is the kinetic energy of turbulence, $S_{i j}$ is the strain rate tensor of the mean flow, and $\delta_{i j}$ is the Kronecker tensor [95]. Under this assumption, Eqs. (1.1) and (1.2) do not have nonzero solutions, so that a correct description of secondary flows is impossible. Thus, the most popular models, such as the Spalart-Allmaras model [98], $k-\varepsilon$ [99], $k-\omega$ [100], or $k-\omega$ SST [101] cannot describe the secondary flow generation [102].

The anisotropy of the Reynolds stress tensor can be taken into account using the corresponding modification of the Boussinesq approximation. An example of this modification was given in [97], where the standard $v^{2}-f$ model [103] based on the assumption on the linear turbulent viscosity was extended with a nonisotropic contribution to the Boussinesq relation. The extended model was successfully tested against an example of lows in pipes of square and triangular cross-sections, for which reasonable agreement with the available data on the turbulent stress distributions and the secondary flow topologies was obtained. It should be noted that the proposed modification is rather specific for particular sets of conditions [96], which can considerably reduce the range of its applicability [95]. The turbulence models resolving the anisotropy, such as the Reynolds stress transport model (RSTM), introduced in [104], represent the most accurate and fundamentally more complete means of closing the Reynolds equations [96]. In those models, a set of the transport equations for individual components of the Reynolds stress tensor is solved, these equations being supplemented with the equations for the turbulence length or temporal scale, which excludes completely the turbulent viscosity hypothesis [95]. The RST models resolve automatically the Reynolds stress anisotropy and are capable to describe the secondary flow formation. The main problem of RSTM consists in the closure of the term governing the pressure deformation and the diffusion term in the Reynolds stress transport equations which are treated in different ways in different 
formulations. It should also be noted that the RST models pertain to the most computationally expensive RANS models, owing to their higher complexity and a greater set of equations necessary for the solution: the RSTM solution requires twice as much time compared with the solution of the nonlinear $k-\varepsilon$ model [95]. The RST approach was first applied to numerical simulation of flow in a square channel [105], the velocity profiles and turbulent characteristics obtained being in reasonable agreement with the experimental data. In [106] developing and developed flows in closed and open channels were studied using the RST model of Launder, Reece, and Rodi (the so-named LRR model). Good agreement of the secondary flows in different configurations was obtained with the experimental data $[1,107]$ and the previous calculations [105, 108]. In the other known RST model, the so-named SSG model [109], an extended quadratic pressure-strain correlation model was used (instead of the linear pressure-strain dependence in LRR [110]). It was shown that the SSG model is superior to the LRR model [109] and is simpler in numerical realization [111]. The SSG model was tested in [112] against an example of flow in a rectangular open channel. The authors reported that the SSG model predicts successfully not only the mean flow but also the distinctive features of the pulsation characteristics. This model was applied in studying the properties of turbulent flows in rectangular [111] and trapezoidal [113] channels, meandering flows in open channels [114], and flows in rectangular open channels of limited depth with bottom vegetation [115].

For the purpose of reducing the computational efforts in solving the transport equations for individual components of the Reynolds stress tensor on the basis of the RST approach, a new class of turbulence models was developed. It uses simplified algebraic relations for calculating the Reynolds stresses, the socalled algebraic stress transport models (ASTM). The first attempt to use ASTM in calculating flows in noncircular pipes was undertaken in [108] on the basis of the AST formulation proposed in [116]. The model ensures an acceptable representation of the secondary flow despite the fact that the turbulent anisotropy is underestimated by an order of magnitude [117, 118]. Moreover, certain coefficients of the model should be so tuned that the secondary motion intensity could diminish down to the lower and, therefore, more physical values. Later, an analogous ASTM realization proposed in [119] was applied in $[120,121]$ for calculating flows in rectangular and elliptical channels. The tendencies of the main stream deformation by the secondary flow obtained in the calculations were correct despite some errors in the details of the mean flow velocity and wall friction distributions. In [122] the ASTM model proposed in [108] was extended with an additional equation for the turbulent kinetic energy with an anisotropic approach to the determination of turbulent viscosity. The model was used in calculating flows in a square pipe and open rectangular channels of different shapes. The authors report on good agreement for all features of the mean and secondary flows, including the modification of turbulent characteristics near the free surface. In the further model modification [24] the contribution of the secondary flow velocity to the calculation of the Reynolds stresses was taken into account (it was neglected [108] or simplified [105, 121] in the previous studies). The calculations were performed for the cases of a square pipe and a rectangular channel with partially rough walls. The comparison with the experiment [15] showed that the model performs better than $[108,122]$ but suffers, nevertheless, from certain underestimation of the primary flow deformation and the secondary flow intensity. A class of model analogous to ASTM was developed from an RST analysis based on the nonlinear strain-stress dependence [123]. This class of models is called nonlinear (or anisotropic) models of turbulent viscosity [95]. The nonlinear realizations of the $k-\varepsilon$ and $k-l$ models proposed in [123] were applied for calculating flows in rectangular straight channels [124] and curvilinear channels [125]. It was shown that, as distinct from their linear counterparts, the nonlinear models are capable to more successfully predict turbulent secondary flows and their effect on the primary flow.

In [126, 127] the nonlinear $k-\varepsilon$ model is compared with the RST-based LRR model [110] for different geometries in configurations of pipes and open channels. Generally, both models are capable to fairly accurately predict the secondary flow formation and its detailed features. However, the author emphasizes that the velocity profile deformation due to the secondary flow is better captured in the RST approach, whereas the secondary flow effect on the wall friction is better reproduced by the nonlinear $k-\varepsilon$ model. The comparison between the results of flow simulation in a trapezoidal channel obtained using the $k-\varepsilon$, $k-\omega$, SST, LRR, and SSG models [128] emphasizes the superiority of the SSG formulation. As might be expected, the linear models of turbulent viscosity do not reproduce the secondary flows. The LRR model presents an incomplete topology of the secondary flow. The SSG model is the unique model demonstrating detailed and accurate secondary flow characteristics. Summarizing the talk it can be said that the RST models ensure the most complete and accurate description of secondary flows within the framework of the RANS modeling. The SSG model gives more accurate results than the LRR model and demonstrates the performance comparable with less computationally expensive models, such as the ASTM model and nonlinear models of turbulent viscosity. 


\section{SUMMARY}

In this review, the studies concerned with Prandtl's secondary flows of the second kind are presented; they appear in turbulent flows under the action of the Reynolds stresses. The secondary flow formation in straight pipes is an interesting manifestation of turbulence. An investigation of the conditions of their generation and the particularities of their manifestation and influence on the main stream is important from both fundamental and applied perspectives. Apart from the review of theoretical, experimental, and numerical studies, in this paper we formulate a principle, which makes it possible to explain and, in certain cases, predict the shape of the secondary flows occurring in smooth pipes with different cross-sections. The reason for the formation of the so-called internal vortices in the corners of junction between solid walls and a free surface is predicted theoretically and explained. A review of investigations of the secondary flows caused by wall roughness inhomogeneities, which has been actively studied in the recent years, is given for the first time. The questions of semiempirical modeling of turbulent flows in the presence of secondary flows are touched upon.

\section{FUNDING}

The study was carried out with the support of the Russian Foundation for Basic Research within the framework of project no. 20-11-50031 using the facilities of the Supercomputer Center of Moscow State University and the Research Center "Kurchatov Institute," http://computing.nrcki.ru/.

\section{DECLARATION OF CONFLICTING INTERESTS}

The Authors declare no potential conflicts of interest with respect to the research, authorship, and/or publication of this article.

\section{OPEN ACCESS}

This article is licensed under a Creative Commons Attribution 4.0 International License, which permits use, sharing, adaptation, distribution and reproduction in any medium or format, as long as you give appropriate credit to the original author(s) and the source, provide a link to the Creative Commons license, and indicate if changes were made. The images or other third party material in this article are included in the article's Creative Commons license, unless indicated otherwise in a credit line to the material. If material is not included in the article's Creative Commons license and your intended use is not permitted by statutory regulation or exceeds the permitted use, you will need to obtain permission directly from the copyright holder. To view a copy of this license, visit http://creativecommons.org/licenses/by/4.0/.

\section{REFERENCES}

1. J. Nikuradse, "Untersuchung über die Geschwindigkeitsverteilung in turbulenten Strömungen," V.D.I. Forschungsheft 70, 1229-1230 (1926).

2. L. Prandtl, "Über die ausgebildete Turbulenz," in: Verhandl. des II. Int. Kongr. für Techn. Mech. (1926), pp. 6275.

3. L. Prandtl, "Turbulent flow," NACA TM-435 (1927).

4. H. Schlichting, Boundary Layer Theory (McGraw-Hill, New York, 1968).

5. J. Nikuradse, "Turbulente Strömung in nicht-kreisförmigen Rohren," Ing.-Arch. 1, 306-332 (1930).

6. L. C. Hoagland, "Fully developed turbulent flow in straight rectangular ducts - secondary flow, its cause and effect on the primary flow," PhD thesis, Department of Mechanical Engineering, Massachusetts Institute of Technology (1960).

7. L. Prandtl, Einführung in die Grundbegriffe der Strömungslehre (Akademische Verlagsgesellschaft, 1931).

8. L. Prandtl, Hydroaeromechanics (Research Center 'Regular and Chaotic Dynamics', Izhevsk, 2000) [in Russian].

9. N. Nikitin and A. Yakhot, "Direct numerical simulation of turbulent flow in ellipticalal ducts," J. Fluid Mech. 532, 141-164 (2005).

10. P. Bradshaw, “Turbulent secondary flows,” Annu. Rev. Fluid Mech. 19(1), 53-74 (1987).

11. V. I. Kornilov, Three-Dimensional Turbulent Near-Wall Flows in Corner Configurations (Siberian Branch of Russian Academy of Sciences, Novosibirsk, 2013) [in Russian].

12. V. I. Kornilov, "Three-dimensional turbulent near-wall flows in streamwise corners: Current state and questions," Progr. Aerospace Sci. 94, 46-81 (2017).

13. L. Prandtl, Essentials of Fluid Dynamics (Hafner, 1952). 
14. J. Hinze, "Secondary currents in wall turbulence," Phys. Fluids (Suppl.) 10, S122-S125 (1967).

15. J. O. Hinze, "Experimental investigation of secondary currents in the turbulent flow through a straight conduit," Appl. Sci. Res. 28, 453-465 (1973).

16. H. A. Einstein and H. Li, "Secondary currents in straight channels," Trans. Amer. Geophys. Union 39(6), 1085-1088 (1958).

17. R. Moissis, "Secondary flow in rectangular ducts," Master's Thesis, Massachusetts Institute of Technology (1957).

18. S. H. Maslen, "Transverse velocities in fully-developed flows," Quart. Appl. Math. 16, 173-175 (1958).

19. E. Brundrett and W. D. Baines, "The production and diffusion of vorticity in duct flow," J. Fluid Mech. 19(3), 375-394 (1964).

20. A. A. Townsend, "Turbulence," in: Handbook of Fluid Dynamics, Ed by V. L. Streeter (McGraw-Hill, New York, 1961).

21. C. G. Speziale, "On turbulent secondary flows in pipes of noncircular cross-section,” Int. J. Engng Sci. 20(7), 863-872 (1982).

22. H. J. Perkins, "The formation of streamwise vorticity in turbulent flow," J. Fluid Mech. 44, 721-740 (1970).

23. F. B. Gessner and J. B. Jones, "On some aspects of fully-developed turbulent flow in rectangular channels," J. Fluid Mech. 23(4), 689-713 (1965).

24. A. O. Demuren and W. Rodi, "Calculation of turbulence-driven secondary motion in non-circular ducts," J. Fluid Mech. 140, 189-222 (1984).

25. R. K. Madabhushi and S. P. Vanka, "Large eddy simulation of turbulence-driven secondary flow in a square duct," Phys. Fluids A 3, 2734-2745 (1991).

26. S. Gavrilakis, "Numerical simulation of low-Reynolds-number turbulent flow through a straight square duct," J. Fluid Mech. 244, 101-129 (1992).

27. A. Huser and S. Biringen, "Direct numerical simulation of turbulent flow in a square duct," J. Fluid Mech. 257, 65-95 (1993).

28. N. V. Nikitin, V. O. Pimanov, and N. V. Popelenskaya, "Mechanism of formation of Prandtl's secondary flows of the second kind," Doklady Physics 64(2), 61-65 (2019).

29. N. V. Nikitin, "Numerical simulation of turbulent flows in a pipe of square cross section," Doklady Physics 42(3), 158-162 (1997).

30. A. Pinelli, M. Uhlmann, A. Sekimoto, and G. Kawahara, "Reynolds number dependence of mean flow structure in square duct turbulence," J. Fluid Mech. 644, 107-122 (2010).

31. M. Kim and D. You, "Reynolds number effect on turbulent secondary flow in a duct," J. Mech. Sci. Technol. 28, 1311-1318 (2014).

32. H. Zhang, F. X. Trias, A. Gorobets, Y. Tan, and A. Oliva, "Direct numerical simulation of a fully developed turbulent square duct flow up to $\operatorname{Re}_{\tau}=1200$," Int. J. Heat Fluid Flow 54, 258-267 (2015).

33. S. Pirozzoli, D. Modesti, P. Orlandi, and F. Grasso, "Turbulence and secondary motions in square duct flow," J. Fluid Mech. 840, 631-655 (2018).

34. N. V. Nikitin and N. V. Popelenskaya, "Main characteristics of secondary turbulent flows in straight pipes," in: Lomonosov Readings. Scientific Conference. Section of Mechanics. April 17-26, 2017. Abstracts of the Reports (Moscow Univ. Press, 2017) [in Russian].

35. J. Ohlsson, P. Schlatter, P. F. Fischer, and D. Henningson, "Direct numerical simulation of separated flow in a three-dimensional diffuser," J. Fluid Mech. 650, 307-318 (2010).

36. H. S. Choi and T. S. Park, "The influence of streamwise vortices on turbulent heat transfer in rectangular ducts with various aspect ratios," Int. J. Heat Fluid Flow 40, 1-14 (2013).

37. R. Vinuesa, A. Noorani, A. Lozano-Duran, G. K. El Khoury, P. Schlatter, P. F. Fischer, and H. M. Nagib, "Aspect ratio effects in turbulent duct flows studied through direct numerical simulation," J. Turbulence 15, 677706 (2014).

38. R. Vinuesa, P. Schlatter, and H. M. Nagib, "On minimum aspect ratio for duct flow facilities and the role of side walls in generating secondary flows," J. Turbulence 16, 588-606 (2015).

39. R. Vinuesa, C. Prus, P. Schlatter, and H. M. Nagib, "Convergence of numerical simulations of turbulent wallbounded flows and mean cross-flow structure of rectangular ducts," Meccanica 51, 3025-3042 (2016).

40. R. Vinuesa, P. Schlatter, and H. M. Nagib, "Secondary flow in turbulent ducts with increasing aspect ratio," Phys. Rev. Fluids 3, 054606 (2018).

41. A. Vidal, R. Vinuesa, P. Schlatter, and H. M. Nagib, "Impact of corner geometry on the secondary flow in turbulent ducts,' in: Proc. 10th Intern. Symp. Turbulence and Shear Flow Phenomena, TSFP-10, Chicago, USA, 2017 (2017).

42. A. Vidal, R. Vinuesa, P. Schlatter, and H. M. Nagib, "Influence of corner geometry on the secondary flow in turbulent square ducts," Int. J. Heat Fluid Flow 67, 69-78 (2017).

FLUID DYNAMICS Vol.56 No.4 2021 
43. A. Vidal, R. Vinuesa, P. Schlatter, and H. M. Nagib, "Turbulent rectangular ducts with minimum secondary flow,” Int. J. Heat Fluid Flow 72, 317-328 (2018).

44. J. Kim, D. Kim, and H. Choi, "An immersed-boundary finite-volume method for simulations of flow in complex geometries," J. Comput. Phys. 171, 132-150 (2001).

45. T. V. Voronova and N. V. Nikitin, "Direct numerical simulation of the turbulent flow in an ellipticalal pipe," Comput. Math. Math. Phys. 46(8), 1378-1386 (2006).

46. T. V. Voronova and N. V. Nikitin, "Results of direct numerical simulation of the turbulent flow in a pipe of ellipticalal cross-section," Fluid Dynamics 42(2), 201-211 (2007).

47. N. Nikitin, "Finite-difference method for incompressible Navier-Stokes equations in arbitrary orthogonal curvilinear coordinates," J. Comput. Phys. 217, 759-781 (2006).

48. S. C. Kacker, "Some aspects of fully developed turbulent flow in non-circular ducts," J. Fluid Mech. 57, 583602 (1973).

49. J. M. Nouri, H. Umur, and J. H. Whitelaw, "Flow of Newtonian and non-Newtonian fluids in concentric and eccentric annuli,” J. Fluid Mech. 253, 617-641 (1993).

50. N. V. Nikitin, "Direct numerical simulation of turbulent flows in eccentric pipes," Comput. Math. Math. Phys. 46(3), 489-504 (2006).

51. N. Nikitin, H. Wang, and S. Chernyshenko, "Turbulent flow and heat transfer in eccentric annulus," J. Fluid Mech. 638, 95-116 (2009).

52. N. Kanaris, X. Albets-Chico, and S. C. Kassinos, "Numerical simulations of turbulent flow in an eccentric annulus of unit eccentricity," in: 9th Intern. Symp. on Turbulence and Shear Flow Phenomena, TSFP 2015. vol. 3, TSFP-9. (2015).

53. N. R. Panchapakesan and P. N. Joubert, "Turbulent boundary layer development along a streamwise edge (Chine) - mean flow," in: 13th Australasian Fluid Mechanics Conference, Monash. (1998), pp. 373-376.

54. N. R. Panchapakesan and P. N. Joubert, "Turbulence measurements in the boundary layer over a streamwise edge (Chine)," in: Turbulence and Shear Flow Phenomena, Santa Barbara (1999).

55. K. A. M. Moinuddin, P. N. Joubert, and M. S. Chong, "Experimental investigation of turbulence-driven secondary motion over a streamwise external corner," J. Fluid Mech. 511, 1-23 (2004).

56. H. Xu and A. Pollard, "Large eddy simulation of turbulent flow in a square annular duct," Phys. Fluids 13(11), 3321-3337 (2001).

57. H. Xu, "Direct numerical simulation of turbulence in a square annular duct," J. Fluid Mech. 621, 23-57 (2009).

58. N. V. Nikitin and N. V. Popelenskaya, "Turbulent flow along an external corner," in: Lomonosov Readings. Scientific Conference. Section of Mechanics. October 2020. Abstracts of the Reports (Moscow Univ. Press, 2020) [in Russian].

59. A. Tominaga, I. Nezu, K. Ezaki, and H. Nakagawa, "Three-dimensional turbulent structure in straight openchannel flows," J. Hydraul. Res. 27, 149-173 (1989).

60. I. Nezu and H. Nakagawa, Turbulence in Open-Channel Flows (Balkema, Rotterdam, 1993).

61. Y. Pan and S. Banerjee, "Numerical investigation of free-surface turbulence in open-channel flows," Phys. Fluids 7, 1649-1664 (1995).

62. I. Albayrak and U. Lemmin, "Secondary currents and corresponding surface velocity patterns in a turbulent open-channel flow over a rough bed," J. Hydraul. Eng. 137(11), 1318-1334 (2011).

63. S.-Q. Yang, S. K. Tan, and X.-K. Wang, "Mechanism of secondary currents in open channel flows," J. Geophys. Res. 117, F04014 (2012).

64. T. Stoesser, R. McSherry, and B. Fraga, "Secondary currents and turbulence over a non-uniformly roughened open-channel bed," Water 7(9), 4896-4913 (2015).

65. I. Nezu. and W. Rodi, "Experimental study on secondary currents in open channels flow," in: Proc. 21st Congress of IAHR. 2. Melbourne. Australia (1985), pp. 19-23.

66. L. M. Grega, T. Wey, R. I. Leighton, and J. C. Neves, "Turbulent mixed-boundary flow in a corner formed by a solid wall and a free-surface," J. Fluid Mech. 294, 17-46 (1995).

67. R. Broglia, A. Pascarelli, and U. Piomelli, "Large-eddy simulations of ducts with a free surface," J. Fluid Mech. 484, 223-253 (2003).

68. W. Anderson, J Barros., K. Christensen, and A. Awasthi, "Numerical and experimental study of mechanisms responsible for turbulent secondary flows in boundary layer flows over spanwise heterogeneous roughness," J. Fluid Mech. 768, 316-347 (2015).

69. O. Marin, R. Vinuesa, A. V. Obabko, and P. Schlatter, "Characterization of the secondary flow in hexagonal ducts," Phys. Fluids 28, 125101 (2016).

70. C. Vanderwel, A. Stroh, J. Kriegseis, B. Frohnapfel, and B. Ganapathisubramani, "The instantaneous structure of secondary flows in turbulent boundary layers," J. Fluid Mech. 862, 845-870 (2019).

71. K. Nagata, J. C. R. Hunt, Y. Sakai, and H. Wong, "Distorted turbulence and secondary flow near right-angled plates," J. Fluid Mech. 668, 446-479 (2011). 
72. E. A. Eichelbrenner and J. H. Preston, "On the role of secondary flow in turbulent boundary layers in corners (and salients),” J. Mécanique 10, 91-112 (1971).

73. F. B. Gessner, "The origin of secondary flow in turbulent flow along a corner," J. Fluid Mech. 58, 1-25 (1973).

74. Dai Yi-Jun and Xu Chun-Xiao, "Wall pressure and secondary-flow origination in a square duct," Phys. Fluids 31, 085104 (2019).

75. N. Nikitin, "Turbulent secondary flows in channels with no-slip and shear-free boundaries," J. Fluid Mech. 917, A24 (2021).

76. D. E. Pivovarov, N. V. Popelenskaya, and N. V. Nikitin, "Direct numerical simulation of turbulent flow over an external corner," in: Abstracts of the Reports Presented to XXIV International Conference 'Nonlinear Problems of Hydrodynamic Stability and Turbulence', Ed by N. V. Nikitin and N. V. Popelenskaya (Moscow Univ. Press, 2020) [in Russian].

77. J Bons., R. Taylor, S. McClain, R. Rivir, "The many faces of turbine surface roughness," in: ASME Turbo Expo 2001: Power for Land, Sea, and Air (2001), pp. V003T01A042-V003T01A042.

78. R. Mejia-Alvarez and K. T. Christensen, "Wall-parallel stereo particle image velocimetry measurements in the roughness sublayer of turbulent flow over-lying highly irregular roughness," Phys. Fluids 25(11), 115109 (2013).

79. J. M. Barros and K. T. Christensen, "Observations of turbulent secondary flows in a rough-wall boundary layer,” J. Fluid Mech. 748, R1 (2014).

80. Z.-Q. Wang and N.-S. Cheng, "Time-mean structure of secondary flows in open channel with longitudinal bedforms," Adv. Water Resources 29(11), 1634-1649 (2006).

81. I. Castro, J. Kim, A. Stroh, and H. Lim, “Channel flow with large longitudinal ribs,” J. Fluid Mech. 915, A92 (2021).

82. D. Chung, J. P. Monty, and N. Hutchins, "Similarity and structure of wall turbulence with lateral wall shear stress variations," J. Fluid Mech. 847, 591-613 (2018).

83. D. Willingham, W. Anderson, K. T. Christensen, and J. M. Barros, "Turbulent boundary layer flow over transverse aerodynamic roughness transitions: Induced mixing and flow characterization," Phys. Fluids 26(2), 025111 (2014).

84. Y. Zheng and W. Anderson, "Flow-roughness heterogeneity: critical obliquity and salient parameters," J. Fluid Mech. 913, A12 (2021).

85. S. Türk, G. Daschiel, A. Stroh, Y. Hasegawa, and B. Frohnapfel, "Turbulent flow over superhydrophobic surfaces with streamwise grooves,” J. Fluid Mech. 747, 186-217 (2014).

86. D. D. Wangsawijaya, R. Baidya, D. Chung, I. Marusic, and N. Hutchins, "The effect of spanwise wavelength of surface heterogeneity on turbulent secondary flows," J. Fluid Mech. 894, A7 (2020).

87. A. Stroh, K. Schäfer, P. Forooghi, and B. Frohnapfel, "Secondary flow and heat transfer in turbulent flow over streamwise ridges," Int. J. Heat Fluid Flow 81, 108518 (2020).

88. D. B. Goldstein and T.-C. Tuan, "Secondary flow induced by riblets," J. Fluid Mech. 363, 115-151 (1998).

89. C. Vanderwel and B. Ganapathisubramani, "Effects of spanwise spacing on large-scale secondary flows in rough-wall turbulent boundary layers,” J. Fluid Mech. 774, 1-12 (2015).

90. T. Medjnoun, C. Vanderwel, and B. Ganapathisubramani, "Characteristics of turbulent boundary layers over smooth surfaces with spanwise heterogeneities," J. Fluid Mech. 838, 516-543 (2018).

91. A. Zampiron, S. Cameron, and V. Nikora, "Secondary currents and very-large-scale motions in open-channel flow over streamwise ridges," J. Fluid Mech. 887, A17 (2020).

92. H. Hwang and J. Lee, "Secondary flows in turbulent boundary layers over longitudinal surface roughness," Phys. Rev. Fluids 3, 014608 (2018).

93. J. Yang and W. Anderson, "Numerical study of turbulent channel flow over surfaces with variable spanwise heterogeneities: Topographically-driven secondary flows affect external-layer similarity of turbulent length scales," Flow, Turbulence, Combustion 100(1), 1-17 (2018).

94. A. Stroh, K. Schäfer, B. Frohnapfel, and P. Forooghi, "Rearrangement of secondary flow over spanwise heterogeneous roughness," J. Fluid Mech. 885, R5 (2020).

95. S. Pope, Turbulent Flows (Cambridge, 2001).

96. M. Leschziner and D. Drikakis, "Turbulence modelling and turbulent-flow computation in aeronautics," Aeronaut. J. 106, 349-384 (2002).

97. R. Pecnik and G. Iaccarino, "Predictions of turbulent secondary flows using the $v^{2}-f$ model," in: $38 t h$ Fluid Dynamics Conference and Exhibit (2008), p. 3852.

98. P. Spalart and S. Allmaras, "A one-equation turbulence model for aerodynamic flows," in: 30th Aerospace Sciences Meeting and Exhibit (1992), p. 439.

99. W. Jones and B. Launder, "The prediction of laminarization with a two-equation model of turbulence," Int. J. Heat Mass Transfer 15(2), 301-314 (1972).

100. D. Wilcox, Turbulence Modeling for CFD, Vol. 2 (DCW Industries, La Canada 1993).

FLUID DYNAMICS $\quad$ Vol. $56 \quad$ No.4 2021 
101. F. Menter, "Two-equation eddy-viscosity turbulence models for engineering applications,” AIAA J. 32(8), 1598-1605 (1994).

102. G. Alfonsi, "Reynolds-averaged Navier-Stokes equations for turbulence modeling,” Appl. Mech. Rev. 62(4), 040802 (2009).

103. P. Durbin, "Near-wall turbulence closure modeling without 'damping functions'," Theor. Comput. Fluid Dyn. 3(1), 1-13 (1991).

104. J. Rotta, "Statistische Theorie nichthomogener Turbulenz," Zeitschrift für Physik 129(6), 547-572 (1951).

105. D. Naot, A. Savit, and M. Wolfshtin, "Numerical calculation of Reynolds stresses in a square duct with secondary flow," Wärme- und Stoffübertragung 7(3), 151-161 (1974).

106. G. Reece, "Development and application of a generalized Reynolds-stress model of turbulence," PhD Thesis, University of London (1977).

107. H. Leutheusser, "Turbulent flow in rectangular ducts," J. Hydraul. Eng. 89(3), 1-19 (1963).

108. B. Launder and W. Ying, "Prediction of flow and heat transfer in ducts of square cross-section,” Proc. Inst. Mechanical Engineers 187(1), 455-461 (1973).

109. C. Speziale, S. Sarkar, and T. Gatski, "Modelling the pressure-strain correlation of turbulence: an invariant dynamical systems approach,” J. Fluid Mech. 227, 245-272 (1991).

110. B. Launder, G. Reece, and W. Rodi, "Progress in the development of a Reynolds-stress turbulence closure," J. Fluid Mech. 68(3), 537-566 (1975).

111. N. de Cacqueray, D. Hargreaves, and H. Morvan, "A computational study of shear stress in smooth rectangular channels,” J. Hydraul. Res. 47(1), 50-57 (2009).

112. H. Kang and S. Choi, "Reynolds stress modelling of rectangular open channel flow," Intern. J. Numer. Methods Fluids 51(11), 1319-1334 (2006).

113. K. Ansari, H. P. Morvan, and D. M. Hargreaves, "Numerical investigation into secondary currents and wall shear in trapezoidal channels,” J. Hydraul. Eng. 137(4), 432-440 (2011).

114. H. Jing, Y. Guo, C. Li, and J. Zhang, "Three-dimensional numerical simulation of compound meandering open channel flow by the Reynolds stress model," Intern. J. Numer. Methods Fluids. 59(8), 927-943 (2009).

115. S. Choi and H. Kang, "Characteristics of mean flow and turbulence statistics of depth-limited flows with submerged vegetation in a rectangular open channel," J. Hydraul. Res. 54(5), 527-540 (2016).

116. K. Hanjalic and B. Launder, "A Reynolds stress model of turbulence and its application to thin shear flows," J. Fluid Mech. 52(4), 609-638 (1972).

117. S. Kacker, "Discussion of 'prediction of flow and heat transfer in ducts of square cross-section'," Proc. Inst. Mechanical Engineers 187, D147-D148 (1973).

118. A. Nakayama, W. Chow, and D. Sharma, "Calculation of fully developed turbulent flows in ducts of arbitrary cross-section,” J. Fluid Mech. 128, 199-217 (1983).

119. D. Tatchell, "Convection processes in confined three-dimensional boundary layers," PhD Thesis. Imperial College London (1975).

120. A. Gosman and C. Rapley, "Fully-developed flow in passages of arbitrary cross-section," Recent Advances in Numerical Methods in Fluids 1, 335-399 (1980).

121. C. Rapley, “The simulation of secondary flow effects in turbulent noncircular passage flows,” Intern. J. Numer. Methods Fluids 2(4), 331-347 (1982).

122. D. Naot and W. Rodi, "Calculation of secondary currents in channel flow," J. Hydraul. Eng. 108(8), 948-968 (1982).

123. C. Speziale, "On nonlinear $k-l$ and $k-\varepsilon$ models of turbulence," J. Fluid Mech. 178, 459-475 (1987).

124. B. Younis and O. Abdellatif, "Modeling sediment transport in rectangular ducts with a two-equation model of turbulence," in: Sediment Transport Modeling (ASCE, 1989), pp. 197-202.

125. N. Hur, S. Thangam, and C. Speziale, "Numerical study of turbulent secondary flows in curved ducts," J. Fluid Eng. 112, 205-211 (1990).

126. D. Cokljat, “Turbulence models for non-circular ducts and channels," PhD Thesis. City University London (1993).

127. D. Cokljat and B. Younis, "Second-order closure study of open-channel flows," J. Hydraul. Eng. 121(2), 94107 (1995).

128. M. Tajnesaie, E. J. Nodoushan, R. Barati, and M. A. Moghadam, "Performance comparison of four turbulence models for modeling of secondary flow cells in simple trapezoidal channels,” J. Hydraul. Eng. 26(2), 187-197 (2020).

Translated by M. Lebedev 